\title{
Dynamic optimization reveals alveolar epithelial cells as key mediators of host defense in invasive aspergillosis
}

\author{
Jan Ewald ${ }^{\dagger, a, *}$, Flora Rivieccio ${ }^{\dagger, b, d}$, Lukáš Radosab ${ }^{b}$ Stefan Schuster ${ }^{a, *}$, Axel A. Brakhage $^{\ddagger}, \mathrm{b}, \mathrm{d}, *$, \\ Christoph Kaleta ${ }^{\ddagger, c}$ \\ ${ }^{a}$ Department of Bioinformatics, Friedrich Schiller University Jena, Jena, Germany \\ ${ }^{b}$ Department of Molecular and Applied Microbiology, Leibniz Institute for Natural Product Research and Infection \\ Biology - Hans Knöll Institute (HKI), Jena, Germany \\ ${ }^{c}$ Research Group Medical Systems Biology, Christian-Albrecht University of Kiel, Kiel, Germany \\ ${ }^{d}$ Department of Microbiology and Molecular Biology, Institute of Microbiology, Friedrich Schiller University Jena, Jena, \\ Germany
}

\begin{abstract}
Aspergillus fumigatus is an important human fungal pathogen and its conidia are constantly inhaled by humans. In immunocompromised individuals, conidia can grow out as hyphae that damage lung epithelium. The resulting invasive aspergillosis is associated with devastating mortality rates. Since infection is a race between the innate immune system and the outgrowth of A. fumigatus conidia, we use dynamic optimization to obtain insight into the recruitment and depletion of alveolar macrophages and neutrophils. Using this model, we obtain key insights into major determinants of infection outcome on host and pathogen side. On the pathogen side, we predict in silico and confirm in vitro that germination speed is a key virulence trait of fungal pathogens due to the vulnerability of conidia against host defense. On the host side, we find that epithelial cells play a so far underappreciated role in fungal clearance and are potent mediators of cytokine release which we confirm ex vivo. Further, our model affirms the importance of neutrophils in invasive aspergillosis and underlines that the role of macrophages remains elusive. We expect that our model will contribute to improvement of treatment protocols by focusing on the critical components of immune response to fungi but also fungal virulence.
\end{abstract}

Keywords: Dynamic optimization, invasive aspergillosis, fungal infection, dynamic modelling, innate immune response, alveolar epithelial cells

\footnotetext{
${ }^{\dagger, \ddagger}$ These authors contributed equally to this work.

*Contact: JE, janewald07@gmail.com; StS, stefan.schu@uni-jena.de; AB, axel.brakhage@leibniz-hki.de
} 


\section{Introduction}

Since we constantly inhale microorganisms, the human lung is an entry point for opportunistic pathogenic microorganisms, like the mould Aspergillus fumigatus [1, 2]. Besides being a saphrophyte involved in the decay of organic matter in soil, $A$. fumigatus possesses virulence characteristics such as small spores (conidia), a fast growth at body temperature and the production of specific proteins, carbohydrates and secondary metabolites allowing for immune evasion [3--7]. These traits enable $A$. fumigatus to reach the lung alveoli and cause an invasive aspergillosis by filamentous growth (hyphae) into the tissue and dissemination into the host [5, 8]. In the immunocompetent host this is prevented by the fast and efficient clearance of conidia by the innate immune system within a few hours [7, 9]. However, once A. fumigatus grows invasively into human tissues facilitated by a suppressed immune system, mortality is very high (30-95\%) due to non-efficient diagnostics and limited treatment options [10-13].

Along with the advances in medical care the number of patients with defects and suppression of their immune system is expected to grow. Major causes for this trend are the increasing number of cancer patients receiving chemotherapy [14, 15], organ transplant recipients [16] or patients with acquired immune deficiency syndrome (AIDS) [17]. Most recently, a high number of COVID19 patients in intensive care units with extensive ventilation has been accompanied by secondary fungal infections due to Aspergillus spp. [13, 18].

The race between fungal growth and host immune response is complex and involves many cells like alveolar epithelial cells (AEC), alveolar macrophages (AM) and recruited neutrophils [7, 9]. To better understand this spatial and dynamic process computational modeling has proven to be of value [19-24]. These models are based on like agent-based modelling or differential equation systems and contribute to a better understanding of the immune response. In particular, the meaning of spatial-dynamics of AM clearing conidia [20, 22, 23, 25] as well as the influence of the initial fungal burden on clearance and persistence of $A$. fumigatus were studied [19]. A major achievement and advantage of in silico models is the integration of existing biological knowledge and the generation of new hypotheses by disclosing knowledge gaps.

Despite extensive modeling and experimental investigations, the relative contribution of individual host immune cell types in invasive aspergillosis remains elusive [8]. For example, AM were identified as phagocytes of conidia [26] and release cytokines upon fungal infection [27]. Yet, AM depletion in mice at early phases of infection showed no effect on mortality while neutrophil depletion was accompanied with low survival rates [28]. Current models and their analysis are based on immune cells and virtually neglect the contribution of AEC to cytokine release and fungal clearance. Experimental data obtained in vitro, however, suggest that AEC can act as potent phagocytes [29] and release a significant amount of cytokines during their interaction with fungal conidia [30]. It has been postulated that AEC represent a 'neglected portal entry of Aspergillus' [7]. Experimental studies exploring their meaning and functions for invasive aspergillosis are difficult to perform due to the multicellular interactions required to evaluate the role of AEC. Therefore, a focus of our presented model here, is to dissect the contributions of AEC and innate immune cells for defense against $A$. fumigatus.

To elucidate the dynamic process of invasive aspergillosis during the first $24 \mathrm{~h}$, we propose a model using dynamic optimization as a mathematical approach. The mathematical concept of a dynamic system described by differential equations and regulated by control variables matches the dynamics of the innate immune response with recruitment and depletion of neutrophils and AM as monocytes. Due to this advantage, dynamic optimization has also been used to model other 


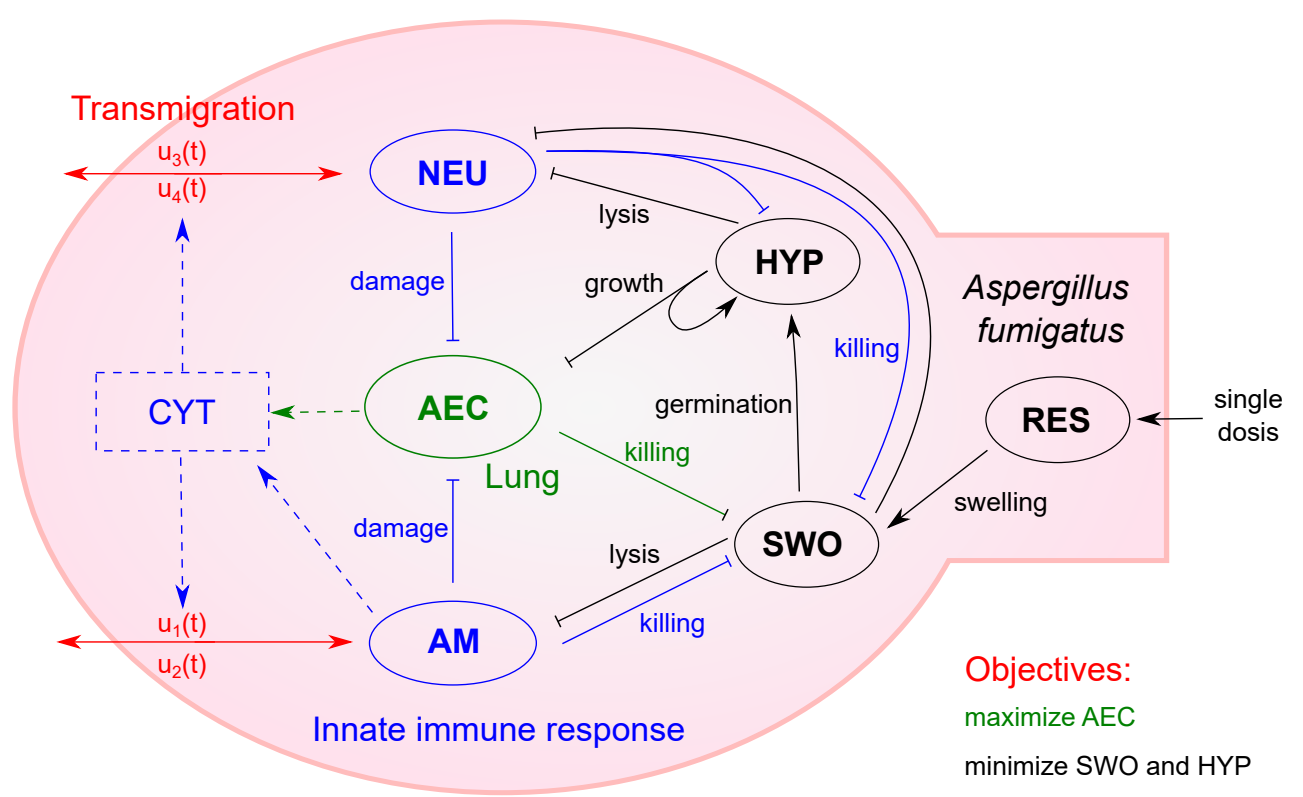

Figure 1: Model of invasive aspergillosis and the innate immune response as dynamic optimization problem. The different fungal growth states (black) including resting conidia (RES), swollen conidia (SWO) and hyphae (HYP) interact with alveolar epithelial cells (AEC, green) as well as with neutrophils (NEU, blue) or alveolar macrophages (AM, blue). Immune cell population is optimized via transmigration (red), which is linked to the cytokine level (CYT, blue), and optimal recruitment and depletion attain the trade-off between pathogen minimization and tissue integrity. Arrow heads indicate a positive interaction and bars show negative interactions.

host-pathogen interactions and immune responses [24, 31, 32] and makes use of the fact that these energy demanding processes are highly optimized during evolution [33]. Our presented model, in addition, not only elucidates the complex recruitment dynamics of immune cells, but we also study the role of AEC during early stages of fungal infection. To proof key variables of the model and thus its validity, major findings like fungal germination and cytokine release were experimentally evaluated.

\section{Results}

\subsection{Model overview}

The aim and scope of our modeling are a better understanding of the decisive parameters and interactions contributing to infection by $A$. fumigatus in the first $24 \mathrm{~h}$. To this end, we model the different growth states of $A$. fumigatus and their interaction with the innate immune response in the lung alveoli during the first hours of infection (see Fig. 1). In addition, we explicitly model AEC as interactive cells and consider a single dosage scenario of fungal conidia exposure. The latter modeling decision enables comparison of results and parameters to experimental animal models, which mainly use single dosage regimes [34, 35].

Our model based on ordinary differential equations (ODE) considers time-dependent transition kinetics to describe the process of swelling and germination of conidia (detailed description in Subsection 4.1). After germination, growth of $A$. fumigatus as hyphae depends on the presence of $A E C$ as resource. Importantly, we accurately model interaction of each cell type with all host 
cell types. Resting conidia are not recognized or killed due to their coating and swollen conidia are phagocytosed and killed by AM, neutrophils and AEC. Since AM and AEC are unable to phagocytose and kill larger hyphae at reasonable rates [36, 37], we only model killing of hyphae by neutrophils.

Host cell dynamics are characterized by damage, host or pathogen mediated, and transmigration of immune cells. In our dynamic optimization model, AEC face lysis by hyphae and damage by activated immune cells. While AM and neutrophils like AEC show cell death upon interaction with fungal cells, their transmigration upon infection is modeled by recruitment and depletion. The maximal rate of recruitment and depletion is linked to the presence of pro-inflammatory cytokines and is optimized in our dynamic optimization approach via the control variables $u_{1-4}$ (see Figure 1 and $c f$. Material and Methods 4.1). In our model we capture the release of cytokines by AM as well as AEC to reveal their respective contribution during infection.

As host objectives we define two main goals, which are optimized during infection. Firstly, active fungal cells (swollen conidia and hyphae) should be minimized at all time points to avert systemic infection. Secondly, unnecessary tissue damage e.g. by hyperinflammation and collateral damage mediated by immune cells must be avoided. The consideration of only one of the objectives leads to undesired dynamics like continued hyphae growth or extensive tissue damage, as shown in Appendix C. Hence, we performed optimization with an equal weighting of both objectives.

\subsection{Parameters and time course of early immune response}

For our dynamic optimization model of the innate immune response during early invasive aspergillosis, reference parameters were estimated based on an extensive study of existing data and literature (see Appendix A). The model focuses on the events in the alveoli during the first $24 \mathrm{~h}$ and we normalize all cell populations per alveolus. Experimental studies and data reveal that the average number of AEC is 11.4 (type I and II combined) in a healthy mouse [38, 39] and on average in every third or fourth alveolus an AM, respectively a neutrophil, is resident [40]. In animal models of invasive aspergillosis typically initial conidia dosages of $10^{5}$ to $10^{7}$ are applied as single dose [7, 35]. Since there are around $2.3 \cdot 10^{6}$ alveoli in a murine lung [39] and not all conidia reach an alveolus, one resting conidium per alveolus relates to a typical fungal burden during experiments. 

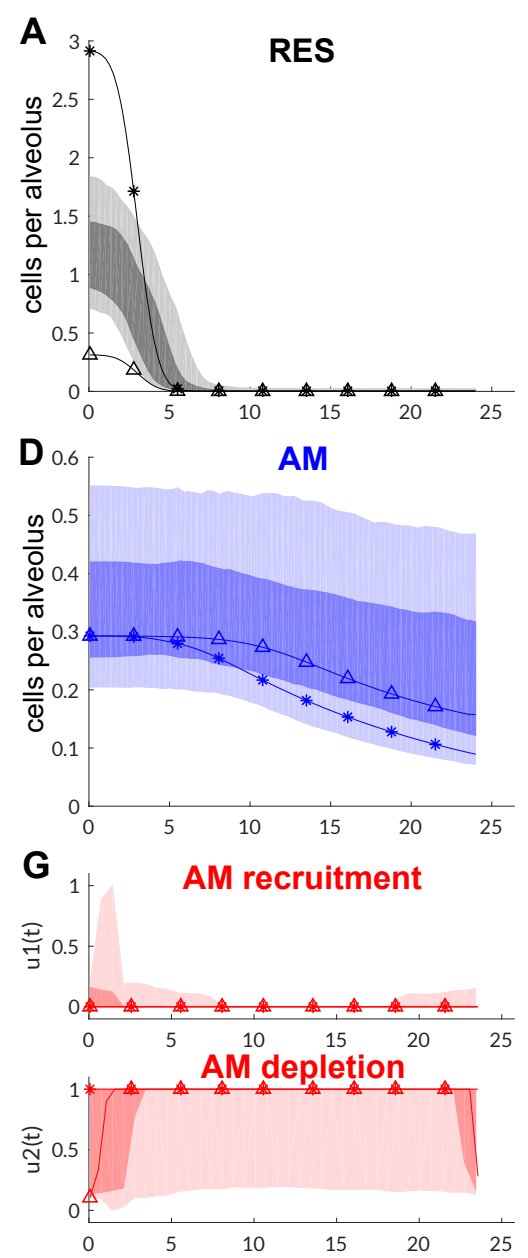

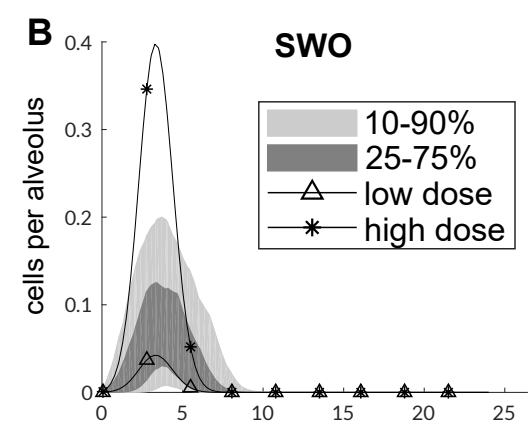

E
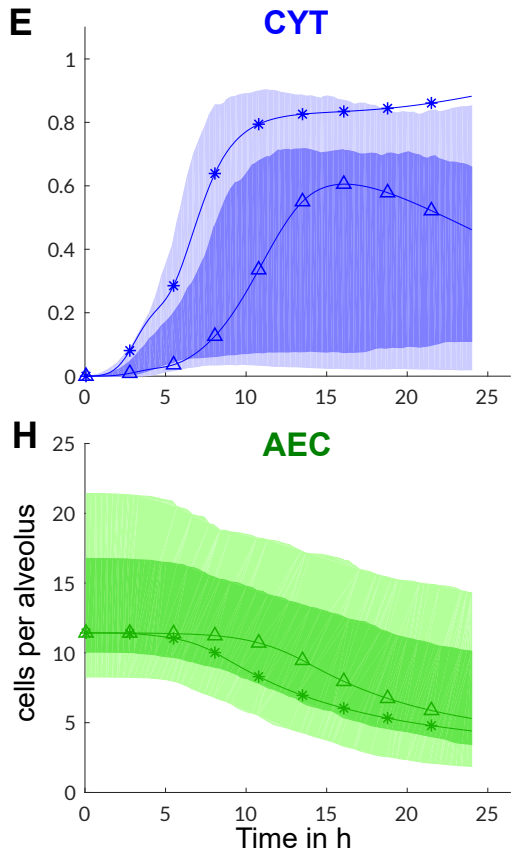
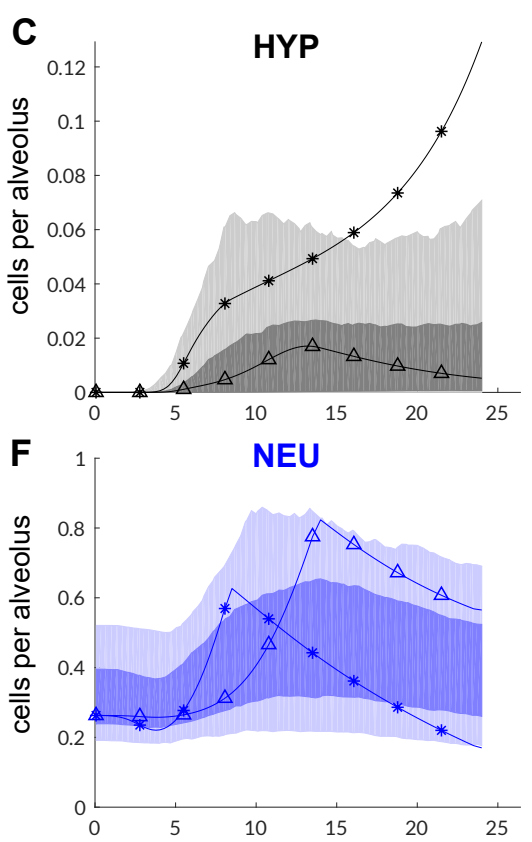

I

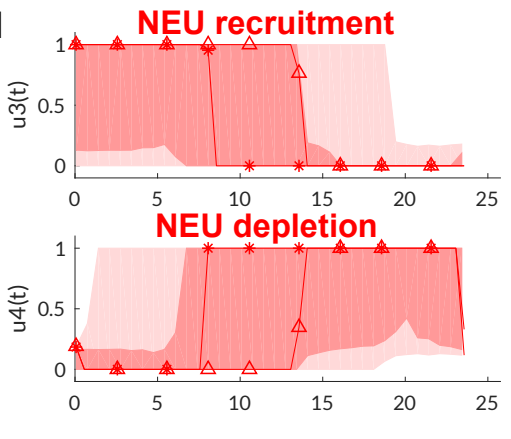

Figure 2: Dynamics of innate immune response of the murine host for varying parameters (shadings) and two dosage scenarios of conidia based on the reference parameter set (lines). A-C Dynamics of fungal cells per alveolus. D, $\mathbf{F}$ show the dynamics of the immune cells per alveolus which are influenced by the optimized recruitment and depletion rates in panels $\mathbf{G}$ and I (rates between 1, maximal, and 0, no recruitment/depletion). The recruitment and depletion potential is dependent on the cytokine level (E). Cytokines are produced by alveolar macrophages (D) and alveolar epithelial cells $(\mathbf{H})$ in response to swollen conidia $(\mathbf{B})$ or hyphae $(\mathbf{C})$. The simulations of 500 parameter sets are depicted with shadings indicating the confidence intervals of time courses.

To elucidate the general pattern of innate immune response during invasive aspergillosis, we simulated 500 randomized parameter sets, where each parameter follows a log-normal distribution with the estimated reference value as the mode (maximum of distribution). The calculated time courses of the dynamic optimization reveal that healthy mice are able to clear even high fungal burden without a complete destruction of the epithelial cell barrier (see Figure 2 and details of solving the dynamic optimization in Subsection 4.2). This is achieved by a rapid cytokine release by $A M$ as well as $A E C$ and recruitment of neutrophils after conidial swelling. After 10 to $15 \mathrm{~h}$, recruitment of neutrophils is stopped and the immune cells are depleted to avoid unnecessary tissue damage. In this regard, our model well reflects experimental observations [41] and depicts the trade-off between pathogen clearance and tissue damage by the innate immune response. In our model, interestingly, AM are not recruited in great quantities and are mostly depleted after germination of conidia. Based on our estimated parameters of the kinetic rates, this is mostly due 
to slower killing of conidia compared to neutrophils and the ability of AEC to release cytokines in great quantities.

\subsection{Parameter sensitivity reveals importance of fungal growth parameters}

To better understand the roles of immune cells and AEC, we performed an analysis of parameter sensitivity and simulated several scenarios to study the effect of immunodeficiencies and conidial dosage.

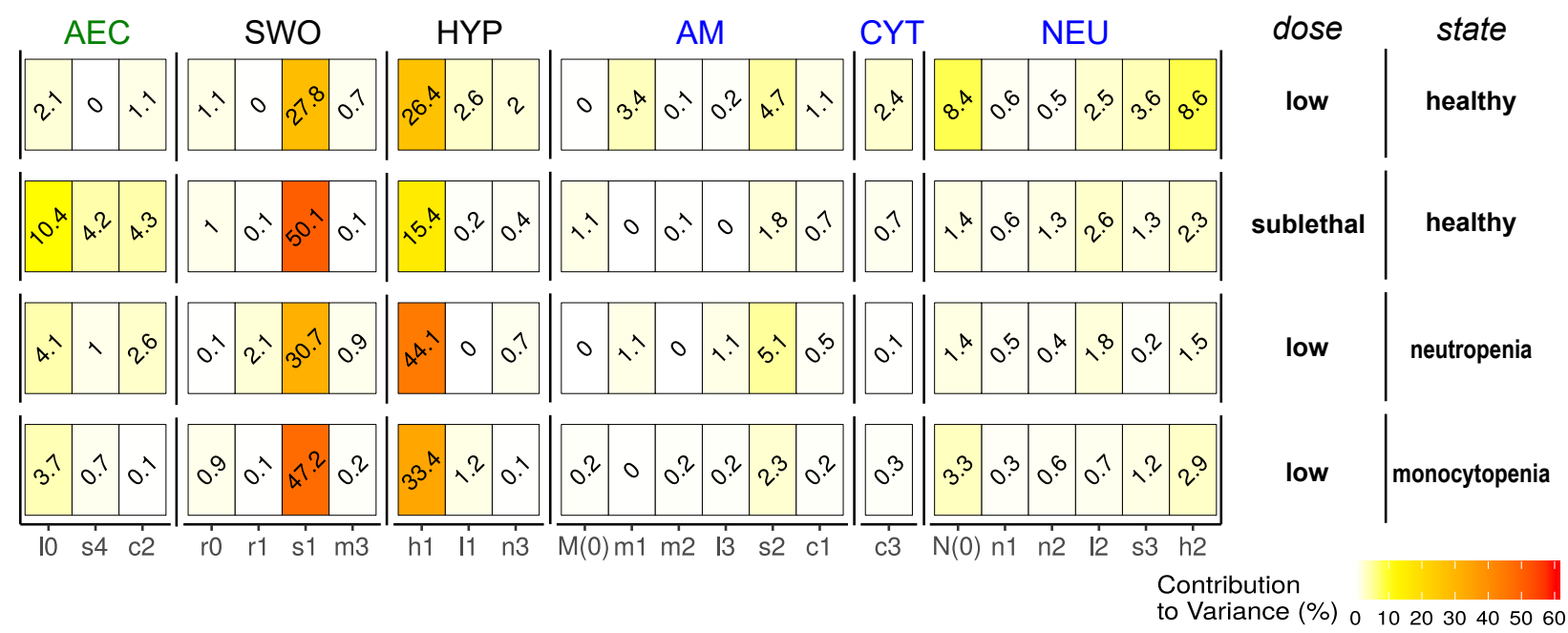

Figure 3: Influence of parameters on the outcome of infection depicted by the contribution to variance (colored from white, yellow to red from no to high influence). This relative contribution is based on a Spearman rank correlation of the parameter value and the objective value of the optimal solution (see Material and Methods 4.2). Parameters are grouped based on their relation to the cell types: alveolar epithelial cells (AEC), resting (RES) or swollen conidia (SWO), hyphae (HYP), alveolar macrophages (AM) and neutrophils (NEU). We list in addition the cytokine (CYT) property $c_{3}$ (decay rate).

We determined decisive parameters for the outcome of infection by calculating the contribution to variance in the objective function of each randomized parameter (see Material and Methods 4.2). Further, we simulated healthy mice and a lack of immune cells as well as the influence of low or high doses of conidia. Across all scenarios we see that the fungal parameters $s_{1}$ (germination time of a swollen conidium) and $h_{1}$ (hyphal growth rate) are most decisive for infection outcome since they explain more than $50 \%$ of the variance in the objective function (see Figure 3). This illustrates that a fast germination of swollen conidia is a strong virulence factor, because it is the most vulnerable growth state of the pathogen. The growth rate of hyphae is in addition crucial in the race between neutrophils (recruitment and hyphal killing) and $A$. fumigatus.

On the host side, we see that the importance of some parameters differs significantly when the initial fungal burden is altered. Interestingly, at lower dosages immune cell parameters like the number of resident neutrophils $N(0)$ or rate of hyphal killing $h_{2}$ are important (see Figure 3). However, at higher dosages parameters of AEC, such as conidia phagocytosis $\left(s_{4}\right)$ or cytokine release $\left(c_{2}\right)$, are more important for the infection outcome and contribute nearly $20 \%$ to the variance in the objective function. The parameter sensitivity and time course of infection indicate that at low dosages the control of hyphal growth is most important, while at high dosages AEC are crucial to lower the number of swollen conidia. These results are noteworthy since parameters related to functions of AM show even combined only a minor influence on the infection outcome $(3.7 \%$ at 
high dosage and $9.5 \%$ at low dose). Typically, AM are extensively studied since they belong to the first line of defense. However, our parameter sensitivity results do not disclose a singular and distinctive role and we therefore performed additional analyses in the following sections.

Further insights can also be gained by studying the changes in parameter influence under scenarios of immunodeficiencies like the lack of monocytes (AM) or neutrophils simulated as reduced, $1 \%$, immune cell populations and recruitment rates (see Figure 3). In both scenarios of immunodeficiencies, fungal virulence factors are even more important for infection outcome. But there are different tendencies as to which factor is more important. During monocytopenia the time span for germination, $s_{1}$, is more important, suggesting that AM are mainly involved in the control of swollen conidia (see Figure 3). In contrast, during neutropenia the hyphal growth rate is more decisive for the infection outcome (see Figure 3) supporting the observation that neutrophils are crucial to prevent filamentous growth.

To support the findings established by modeling, we performed an experimental investigation of fungal virulence parameters by the comparison of different Aspergillus species. As revealed by the parameter sensitivity analysis, a key virulence factor is a fast germination to minimize the time period of the vulnerable swollen-conidia state. The common species $A$. fumigatus, $A$. nidulans, A. niger and $A$. terreus show differences in their germination kinetics at $37^{\circ} \mathrm{C}$, where $A$. nidulans is fastest, $A$. fumigatus as well as $A$. niger are around $1-2 \mathrm{~h}$ slower and $A$. terreus is by far the slowest with an average germination time of $>24 h$ (see Figure $4 \mathrm{~A}$ and $\mathrm{B}$ ). Our model predicts here a non-linear but distinctive relationship between the germination time and epithelial damage after 24h (see Figure 4C). It suggests that virulence expressed as epithelial damage is strongly reduced if the germination time is longer than $10 \mathrm{~h}$. 


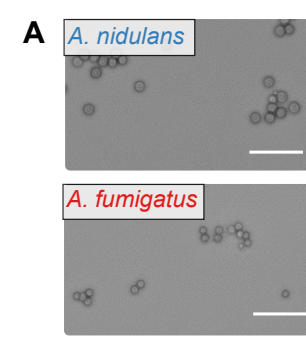

$2 \mathrm{~h}$

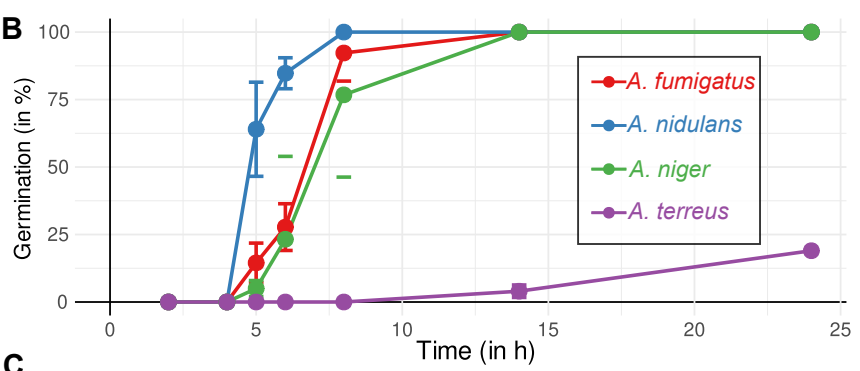

。

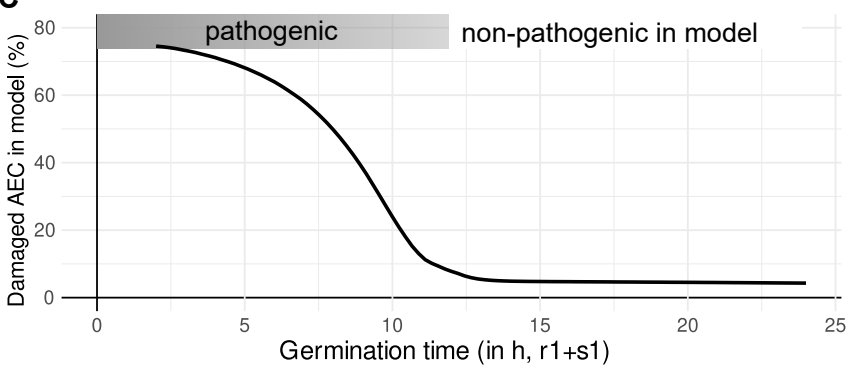

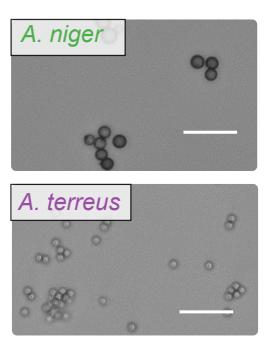

$2 \mathrm{~h}$

D

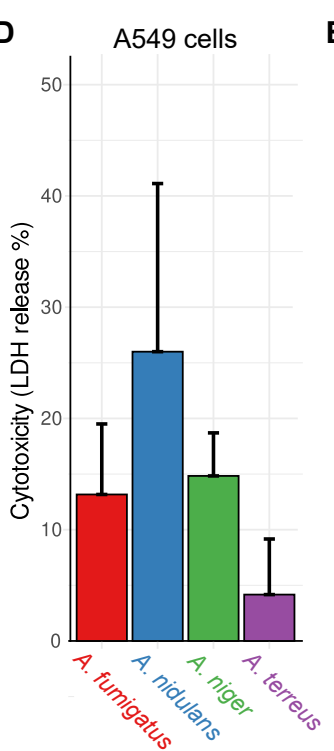

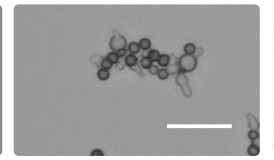
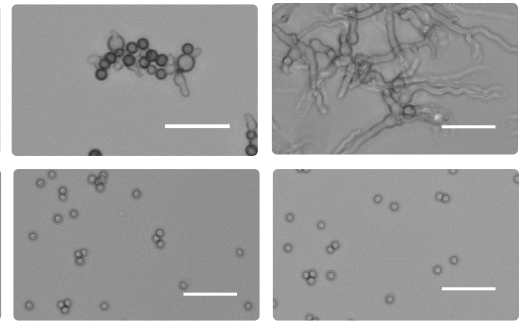

$6 \mathrm{~h}$

E

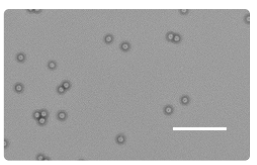

$8 h$

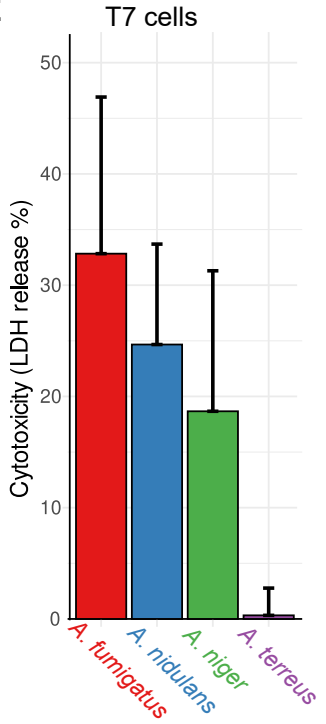

Figure 4: Germination kinetics of Aspergillus spp. and its impact on epithelial cytotoxicity. A Microscopic images (white scale bar, 20 $\mu \mathrm{m}$ ) of germination assays on RPMI medium to automatically count and derive germination kinetics as shown in B for the species A. fumigatus (red), A. nidulans (blue), A. niger (green) and A. terreus (purple). C the relation of germination time and damage of AEC after 24h in the dynamic optimization model and parameter range with expected pathogenicity. Model prediction in $\mathbf{C}$ is compared to experimental cytotoxicity of Aspergillus spp. by lactatedehydrogenase (LDH) release measurements after 24h co-incubation with human A549 epithelial cells in D and with murine T7 epithelial cells in $\mathbf{E}$.

Strikingly, in an experimental set-up where human (A549 cell line) and murine (T7 cell line) lung AEC are co-incubated with Aspergillus spp. for 24h, only those species with a fast germination show a pronounced host cell damage (see Figure $4 \mathrm{D}$ and E). Moreover, cytotoxicity against human cells coincides with germination speed and strongly supports model prediction. However, we observed a higher cytotoxicity of $A$. fumigatus against murine AEC while other species show comparable results (see Figure $4 \mathrm{E}$ ). This underlines the importance of further investigations to understand differences between the human host and rodent model organisms. Further, it suggests an avoidance of elevated epithelial damage by $A$. fumigatus and its ability to hide and escape in AEC during the human immune response.

The accordance of modeling prediction with experimental data on germination as well as cytotoxicity shows that our dynamic optimization approach is able to identify key parameters of invasive aspergillosis. However, since A. fumigatus is the most common cause of invasive aspergillosis [10] in spite of its slightly slower germination than $A$. nidulans, the interaction of fungal pathogens with host cells and parameters defining this process represents additional decisive factors for infection outcome. 
To this end, in the following part the specific roles and functions of host cells are investigated by modeling and supported by experimental investigations.

\subsection{Neutrophils and epithelial cells primarily promote fungal clearance and cytokine release}

The advantage of our model is that we can suppress a host cell population or function in silico to study their role and importance for infection outcome. This way we can simulate animal models with immunodeficiencies like neutropenia which is triggered by usage of cyclophosphamide and cortisone acetate in murine models of invasive aspergillosis [42]. The analysis of parameter sensitivity provides a global overview of the correlation between infection parameters and infection outcome. However, the causality of parameter influence is not explained. To this end, we performed additional in silico experiments with varying conidial dosages to understand and link host cell functions to infection outcome.
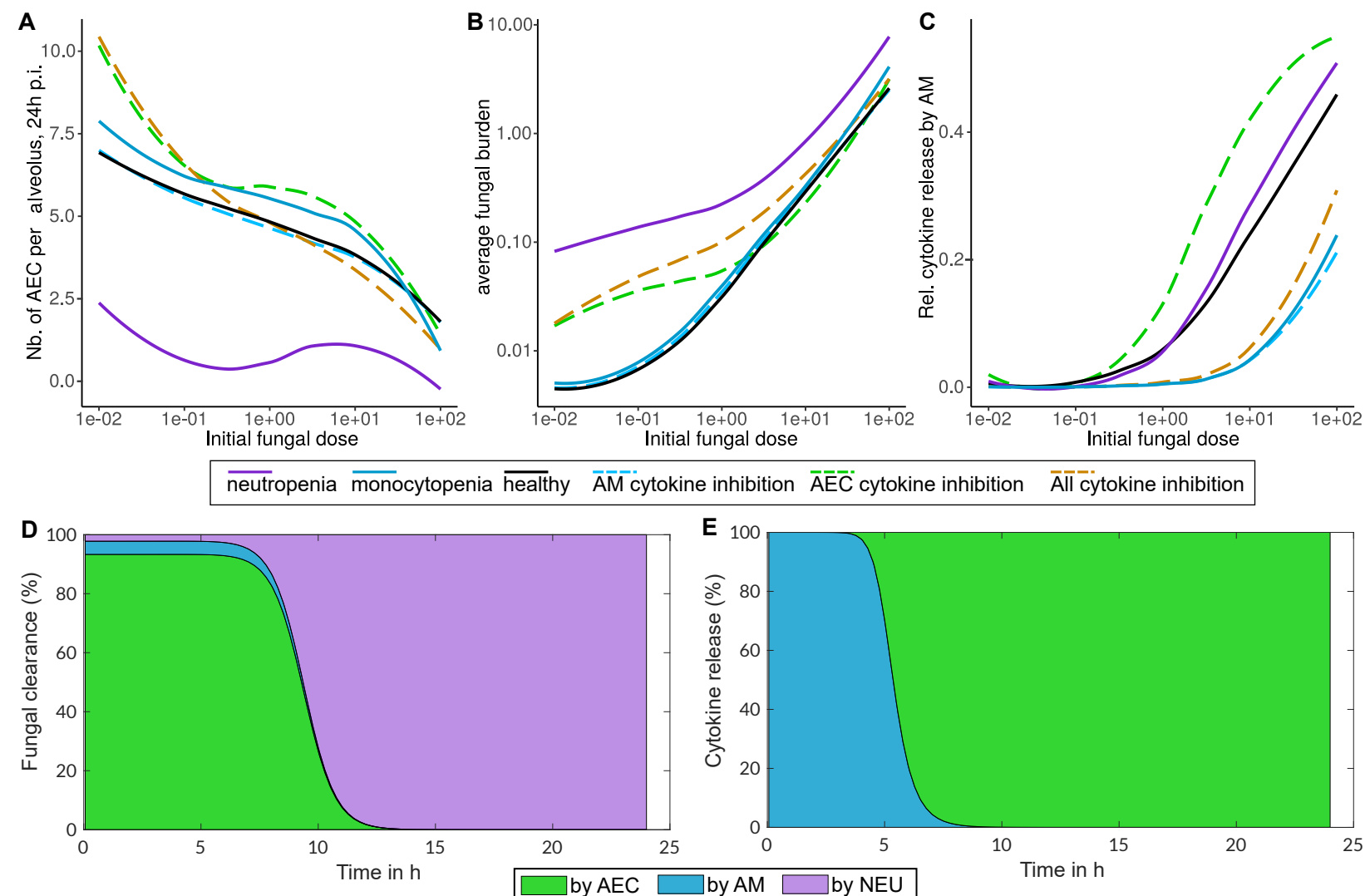

Figure 5: Relative contribution of host cells during infection. A-C Dose-response-curves with varying conidia dosages ( $\mathrm{x}$-axis) and the remaining number of epithelial cells at the end of simulation in $\mathbf{A}$ or the average fungal burden during simulation in $\mathbf{B}$. The relative contribution of alveolar macrophages to cytokine release depending on the initial conidia dosage in $\mathbf{C}$. Response curves were determined for the disturbances: lack of immune cells (1\% neutrophils, solid violet; $1 \%$ macrophages solid light blue) and cytokine release inhibition (1\% of cytokine release by alveolar macrophages (AM, dashed light blue), $1 \%$ release by alveolar epithelial cells (AEC, dashed green) or $1 \%$ release from both (dashed brown). D, E Role of macrophages (AM), neutrophils (NEU) and epithelial cells (AEC) in fungal clearance $\mathbf{A}$ and cytokine release $\mathbf{B}$ in the dynamic optimization model over time as percentage of total at each time point. Fungal clearance is calculated as the sum of killing all fungal cell types (conidia and hyphae).

The conidia dose-response-curves clearly show that lack of neutrophils heavily worsens infection outcome across all dosage scenarios and host damage is barely dose-dependent (see 
Figures $5 \mathrm{~A}$ and $\mathrm{B})$. Further, both objectives, i.e., the preservation of tissue and reduction of the fungal burden, are more impaired than by all other deficiency scenarios including lack of AM or an inhibition of cytokine release. Interestingly, lack of AM or an inhibition of cytokine release by AM show no major differences in the infection outcome compared to the reference parameter set of healthy mice (see Figures $5 \mathrm{~A}$ and $\mathrm{B}$ ). The optimization results for the inhibition of cytokine release by $A E C$ or by both, AEC and AM, indicate a shift in the immune objective at low conidia dosage. In comparison to healthy mice, lung tissue is more preserved at low conidia concentrations, but with the drawback of a higher average of fungal burden during the simulated time of infection.

In our model AM and AEC exhibit overlapping functions and duties during infection. Both are able to clear swollen conidia and to release cytokines for immune cell recruitment. However, their contribution is different depending on the initial fungal burden and during the dynamic stages of infection. When the dosage of conidia is low, the relative contribution of AM to cytokine production is nearly zero and most importantly, germination and hyphal growth are recognized by AEC (see Figure $5 \mathrm{C}$ ). The relative contribution to cytokine release by AM rises to $>40 \%$ at very high conidia concentrations (see $5 \mathrm{C}$ ) and indicates that $\mathrm{AM}$ are more important when a high number of swollen conidia is present (10-100 spores per alveolus).

This finding is supported by the relative contribution of each cell type to fungal clearance and cytokine release over the time course of infection (see Figure 5D). During infection, according to our model, AM are responsible for early recognition of conidia and AEC are the main cytokine releasing cells after germination. However, the low quantities of AM compared to AEC, lead to a much higher cytokine release by $A E C$ compared to $A M$ in absolute and relative terms during infection (see Figure $5 \mathrm{E}$ ).

The prominent role of AEC in pro-inflammatory cytokine production upon fungal infection is not captured by other models of invasive aspergillosis and indicates an underestimated importance of these cells for the immune response. To quantify and support findings predicted by our model, ex vivo experiments with murine cells were performed and pro-inflammatory cytokines (TNF)- $\alpha$ and (IL)- 6 were measured upon stimulation with $A$. fumigatus by enzyme-linked immunosorbent assay (ELISA). These cytokines were selected because of their prominent role during invasive aspergillosis in mice [41, 43-45]. Whereas AM mainly produce (TNF)- $\alpha$ after $6 \mathrm{~h}$ of infection with conidia, murine AEC mainly produce (IL)-6 after 10h (see Figure 6A). At similar cell counts AM produce earlier (6h versus 10h), but less in absolute terms in comparison to AEC when excess release upon conidial challenge of both cytokines, (TNF)- $\alpha$ and (IL)-6, is combined (see Figure 6B). Considering the higher number of $A E C$ than $A M$ in an alveolus, these experimental findings strongly support our model that AEC are important mediators of the immune response.

Moreover, our model reveals that AEC are important phagocytes and are predicted to be the main cell type clearing fungal spores in the first hours of infection (see Figure 5D). Nevertheless, after germination neutrophils are decisive over fungal clearance by killing hyphae. These findings highlight that alveolar epithelial fulfill a major role in fungal clearance but also in immune cell recruitment by cytokine release.

\section{Discussion}

In this study we present a unique model using the approach of dynamic optimization to study the innate immune response during invasive aspergillosis. The model's parameterization builds on an extensive literature search and manual curation, which ensures a proper simulation of the infection dynamics. Main and distinctive features of the model are the optimization of immune 

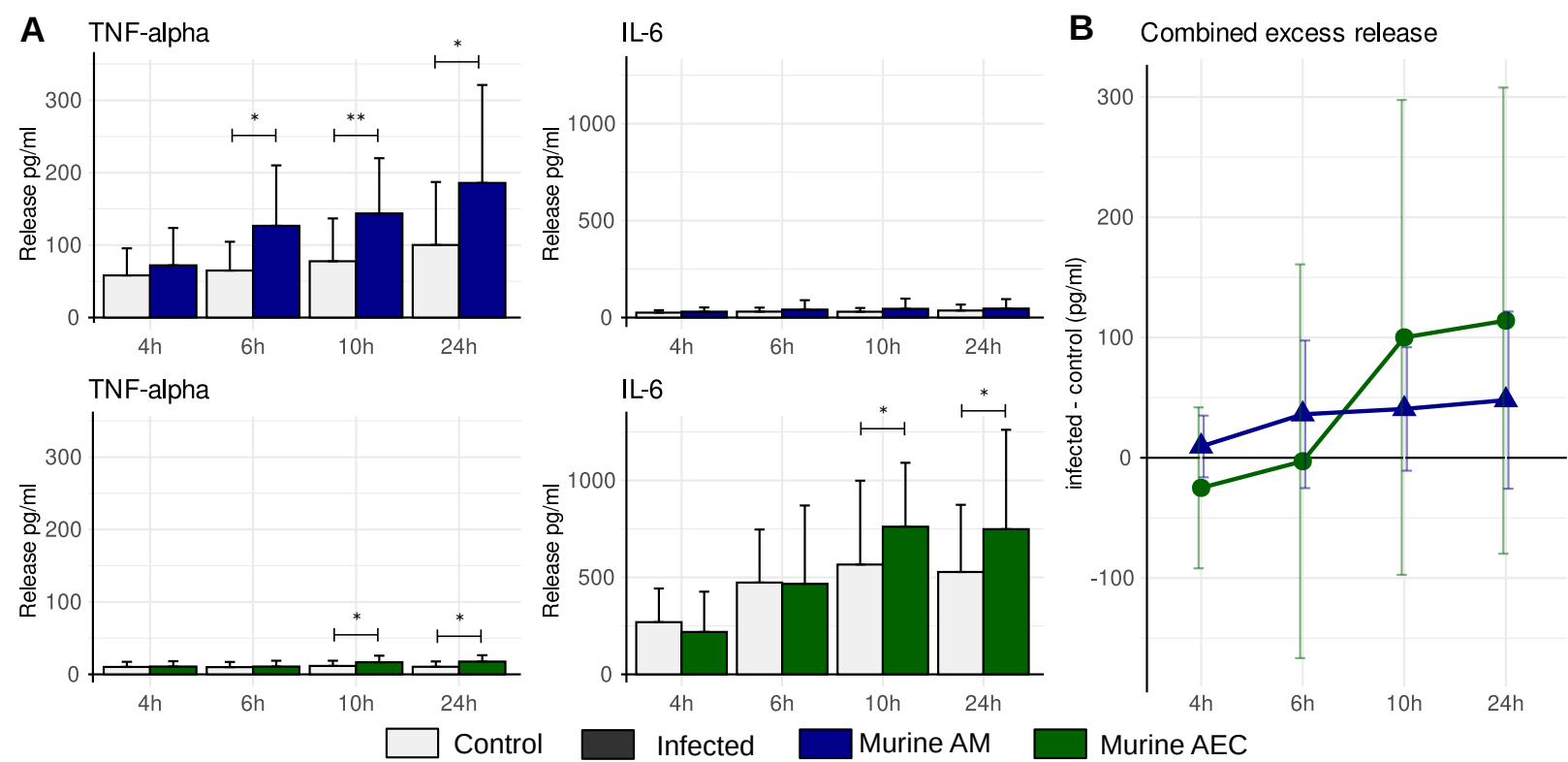

Figure 6: Ex vivo cytokine release of isolated murine alveolar macrophages (blue) and epithelial cells (green) upon infection with $A$. fumigatus. A Release of (TNF)- $\alpha$ and (IL)-6 over time in infected and control cells measured by ELISA. Significant differences between control and infected cells were determined by a two-tailed and paired t-test indicated by $*(p<0.05)$ and $* *(p<0.01)$. B Combined cytokine release of both (TNF)- $\alpha$ and (IL)- 6 in excess over spontaneous release over time to depict contribution during early and later stages of fungal infection.

cell recruitment depicting the trade-off between tissue integrity and pathogen clearance (dynamic optimization) as well as the active role of lung AEC in the immune response.

The simulations and analysis of our model revealed key parameters and insights into the roles of host cells during the innate immune response against $A$. fumigatus and other Aspergilli. We identified the state 'swollen conidia' as most vulnerable for host defense. Hence, its time span is minimized by the fungus to escape phagocytosis and, subsequently, outcompete host cells by fast growth of hyphae. While this was indicated by experimental studies linking fungal traits with clinical observations [46, 47], we here report a quantification of this relationship. Further, we provide experimental evidence by the characterization of different Aspergillus spp. and their germination kinetics, as well as their cytotoxicity against human and murine AEC. Interestingly, in our and other studies $A$. nidulans shows fastest germination and a high damage to AEC. Since A. nidulans is not the leading cause of invasive aspergillosis in humans [48], it is likely that this species lacks immune evasion mechanisms in comparison to A. fumigatus [36]. However, infection by $A$. nidulans is frequently observed in chronic granulomatous disease where the lack of a fully functional NADPH-oxidases strongly limits the capability of host immune cells [49, 50]. Hence, important fungal virulence traits of $A$. fumigatus are presumably linked to an efficient escape or inhibition of phagocytosis in comparison to $A$. nidulans. This conclusion is supported by the novel observation that only $A$. fumigatus shows a stronger cytotoxicity against murine cells in comparison to human AEC (see Figure $4 \mathrm{D}$ and $\mathrm{E}$ ). While this indicates an adaption of $A$. fumigatus to hide and reside in human AEC from other immune cells, further investigation is necessary to understand the differences between the human host and rodent model organisms.

As crucial host parameters for infection outcome we reaffirmed the importance of neutrophils to control filamentous growth of $A$. fumigatus which underlines that dysfunctional hyphal killing or 
neutropenia are major risk factors for invasive aspergillosis [28, 51]. In addition to immune effector cells, we found that lung AEC make an important part of the immune response by contributing extensively to phagocytosis of conidia and cytokine release in response to fungal germination. While this finding was partially observed and suggested in previous studies, we present here the first model which quantifies the role of AEC. Together with experimental measurements that compare the cytokine release of $A M$ and $A E C$, we conclude that the role of $A E C$ is underestimated. This conclusion is largely based on the observation that the number of AEC per alveolus is higher than the number of AM or neutrophils at early stages of infections, while phagocytosis rates and cytokine release are comparable or even higher than observed with AM (see Figure 6).

Despite their detailed investigation by experimental studies and computational models [23, 52], our results suggest that $A M$ are involved in early recognition of swollen conidia and contribute less to fungal clearance than neutrophils and AEC. We observe a slightly higher importance of AM in low dose scenarios that indicates the importance of dosage in animal models of invasive aspergillosis to ensure transferability of results. However, our results are in line with the observation that in murine infection models neutrophil depletion, but not AM depletion, increases mortality rates [28].

Since our model focuses on the innate immune response and is a simplification of complex interactions during fungal infection, additional functions of AM are potentially not represented in the model. Such functions of AM involve e.g. the balance of pro- and anti-inflammatory signaling as well as the support of tissue repair [53, 54]. Further, AM link innate immunity to the adaptive immune system and are important versatile cells to maximize the robustness of the immune system [55, 56].

Additional in silico and in vivo studies are needed to fully understand the roles of AM as well as lung AEC during invasive aspergillosis. Such models and the model presented here are very valuable to identify new treatment approaches and to determine optimal treatment strategies by a combination of approaches. Dynamic optimization has been previously suggested and applied to calculate an optimal time-course of treatment protocols to combat infections by determining an optimal usage of antimicrobials and therapies boosting the immune response [57]. For such purposes, our model provides an excellent starting point to identify time-optimal treatment strategies of invasive aspergillosis.

\section{Material and methods}

\subsection{Model formulation}

We consider three different states, resting and swollen conidia (spores) as well as hyphae (filaments). Resting conidia are administered to the host's lung at varying quantity $R_{0}$. After $3-4 \mathrm{~h}$ conidia will swell in lung alveoli and can be recognized and phagocytosed by host cells [58]. To correctly include the time needed for swelling of conidia, we model the swelling rate as a normal distribution with the mean at time point $r_{1}=4 \mathrm{~h}$ and variance $1 \mathrm{~h}$ :

$$
\dot{R}=\underbrace{-R_{0} f_{n}\left(t \mid r_{1}, 1 h\right)}_{\text {swelling }}, \quad f_{n}\left(t \mid \mu, \sigma^{2}\right)=\frac{1}{\sqrt{2 \pi \sigma^{2}}} e^{-\frac{(t-\mu)^{2}}{2 \sigma^{2}}}
$$

Subsequent to swelling, conidia germinate after an additional delay of $s_{1}=3 h$ to hyphae [58, 59]. The phagocytosis by immune as well as AEC is modeled by simple bilinear terms with 
the specific rates $\left(s_{2-4}\right)$ :

$$
\dot{S}=\underbrace{R_{0} f_{n}\left(t \mid r_{1}, 1 h\right)}_{\text {swelling }} \underbrace{-S f_{n}\left(t \mid r_{1}+s_{1}, 1 h\right)}_{\text {germination }} \underbrace{-S\left(s_{2} M+s_{3} N+s_{4} L\right)}_{\text {phagocytosis }} .
$$

In the multicellular growth state of hyphae the number of $A$. fumigatus cells depends on the rate of germination as well as the growth rate $\left(h_{1}\right)$ of hyphae, which we link to the presence of AEC as a resource for growth. We model killing of hyphae by neutrophils and obtain the following description of hyphal dynamics:

$$
\dot{H}=\underbrace{S f_{n}\left(t \mid r_{1}+s_{1}, 1 h\right)}_{\text {germination }} \underbrace{+h_{1} \frac{L}{L_{0}} H}_{\text {growth }} \underbrace{-h_{2} H N}_{\text {killing }} .
$$

The number of $A E C$ is influenced in our model by the lysis induced by fungal hyphae and the damage originating from active AM and neutrophils. The tissue damage by immune cells is often ignored in computational models, but is crucial to reflect recruitment and depletion of immune cells [60]. To this end, we further connect tissue damage with the pro-inflammatory cytokine level $C$ :

$$
\dot{L}=\underbrace{-l_{1} L H}_{\text {dissemination }} \underbrace{-L C\left(l_{2} N+l_{3} M\right)}_{\text {tissue damage }} .
$$

AM are of relevance for recognition and phagocytosis of swollen conidia. In addition to tissueresident cells, more AM are recruited by transmigration and differentiation of monocytes which circulate in the blood. We assume that this recruitment has a maximal rate of $m_{1}$ cells per hour and depends on the pro-inflammatory cytokine level $C$ and macrophage recruitment is further optimized via the control variable $u_{1}(t)$. This time dependent control variable is optimized by dynamic optimization and can vary between 0 (no macrophage recruitment) to 1 (maximal recruitment) at each time point. In a similar way active depletion or deactivation of AM is modeled $\left(u_{2}(t)\right)$. Lastly, the number of AM also depends on the lysis initiated by germinating conidia and therefore we model macrophage dynamics as:

$$
\dot{M}=\underbrace{m_{1} C u_{1}}_{\text {recruitment }} \underbrace{-m_{2}(C) u_{2}}_{\text {depletion }} \underbrace{-m_{3} M\left(S f_{n}\left(t \mid r_{1}+s_{1}, 1 h\right)\right)}_{\text {lysis by germinating conidia }} .
$$

An important role of $A M$ is the release of pro-inflammatory cytokines in response to fungal cells. However, AEC in addition release cytokines and mediate neutrophil recruitment. Thus, we model the dynamics of a pro-inflammatory cytokine level ranging from 0 (no inflammation) to 1 (maximum inflammation) as follows:

$$
\dot{C}=\underbrace{c_{1}(S+H) M(1-C)}_{\text {inflammation by AMs }} \underbrace{+c_{2} H L(1-C)}_{\text {inflammation by AECs }} \underbrace{-c_{3} C}_{\text {decay }} .
$$

It is important to note that based on experimental observations, AEC start releasing cytokines only after conidia had germinated [30] and AM already in response to swollen conidia [7]. 
Although neutrophils also reside in lung tissue, they are recruited in large quantities from the blood in response to a fungal infection by pro-inflammatory cytokines like interleukin 8 [30]. The kinetics of a neutrophil population is described by:

$$
\dot{N}=\underbrace{n_{1} C u_{3}}_{\text {recruitment }} \underbrace{-n_{2}(C) u_{4}}_{\text {depletion }} \underbrace{-n_{3} N(S+H)}_{\text {lysis by fungi }}
$$

The kinetic parameters are cumbersome to be determined due to the inclusion of optimal control variables and experimental inaccessibility of in vivo infection parameters. Nevertheless, we carefully and extensively reviewed published experiments to estimate each parameter (see Appendix A). Parameters and cell numbers are determined per murine alveolus.

The above described ODE system and parameters are available as SBML file in Appendix B and are stored in the database BioModels [61] under the accession MODEL2105110001.

\section{Constraints and objective of optimization problem}

To determine the optimal innate immune response during invasive aspergillosis, the above described dynamic system has to fulfill the following constraints and objectives. Intuitively, state variables describing cell numbers are positive, pro-inflammatory cytokine level as well as control variables describing recruitment and depletion of immune cells range between 0 and 1 :

$$
0 \leq\left(\begin{array}{c|l}
L(t) \\
R(t) \\
S(t) \\
H(t) \\
M(t) \\
N(t)
\end{array}\right) \begin{aligned}
& \text { alveolar epithelial cells } \\
& \text { resting conidia } \\
& \text { swollen conidia } \\
& \text { hyphae }
\end{aligned} \quad, \quad \underbrace{0 \leq C(t) \leq 1}_{\text {cytokines }}, \quad \underbrace{0 \leq u_{1-4}(t) \leq 1}_{\text {transmigration }}
$$

As objectives we define two main goals of the host organism. Firstly, active fungal cells (swollen conidia and hyphae) should be minimized at all time points to avert systemic infection. Secondly, unnecessary tissue damage e.g. by hyperinflammation and collateral damage mediated by immune cells must be avoided. We formalize as objective function:

$$
F=\min _{u_{1-4}} \int_{0}^{T_{\max }}\left(o_{2} \cdot \frac{S(t)}{r_{0}}+o_{3} \cdot \frac{H(t)}{r_{0}}-o_{1} \cdot \frac{L(t)}{l_{0}}\right) d t .
$$

The normalization to the initial cell numbers and equal weighting $o_{1-3}=0.5$ ensures a balanced and biological meaningful optimization result. As shown in Appendix C, considering only one of the objectives leads to undesired dynamics like continued hyphae growth or extensive tissue damage.

\subsection{Solving the optimization problem and parameter sensitivity}

The resulting dynamic optimization problem with continuous state and control variables was solved by a quasi-sequential approach established and implemented by Bartl et al. [62]. This gradient-based method has proven its capability in several previous applications to biological systems [24, 63-66] and ensures fast and robust calculation of the optimal control. To avoid local optima, we perform for each parameter set at least 100 randomizations of the initial solution, which is used to start the optimization process. 
To determine the sensitivity of parameters, we sampled 500 parameter sets according to a log-normal distribution, where for each parameter the respective mode (maximum of the density function) corresponds to the literature-based parameter value (see Appendix A). The parameter sensitivity is expressed as the contribution to variance [67] and is based on the Spearman correlation $r h o(p)$ between the parameter value $p$ and the objective function value:

$$
c t v_{p}=\frac{r h o(p)^{2}}{\sum_{p} r h o(p)^{2}} .
$$

Our model enables the exploration of different scenarios like different conidia dosages and immunodeficiencies. For mice we use as initial conidia burden a dosage of 1 conidium per alveolus as 'sublethal' and in low dose scenarios 0.1 which are comparable to in vivo experiments. A lack of immune cells like neutropenia or monocytopenia (lack of AM) is simulated by a reduced (1\%) initial cell number and rate of recruitment. In a similar way, cytokine release inhibition is simulated by reducing the rates to $1 \%$ of the reference value.

\subsection{Experimental evaluation of fungal and host infection parameters}

\section{Fungal strains and cultivation}

Aspergillus fumigatus CEA10, Aspergillus nidulans FGSC A4, Aspergillus niger ATCC 1015 and Aspergillus terreus SBUG 844 were grown on Aspergillus minimal medium (AMM; containing $70 \mathrm{mM} \mathrm{NaNO}_{3}, 11.2 \mathrm{mM} \mathrm{KH}_{2} \mathrm{PO}_{4}, 7 \mathrm{mM} \mathrm{KCl}, 2 \mathrm{mM} \mathrm{MgSO}$, and $1^{\mu L} / \mathrm{mL}_{\mathrm{L}}$ trace element solution at $\mathrm{pH}$ 6.5) and agar plates with $1 \%(\mathrm{w} / \mathrm{v})$ glucose for 5 days at $37^{\circ} \mathrm{C}$. The trace element solution was composed of $1 \mathrm{~g} \mathrm{FeSO} \cdot \cdot 7 \mathrm{H}_{2} \mathrm{O}, 8.8 \mathrm{~g} \mathrm{ZnSO} \cdot 7 \mathrm{H}_{2} \mathrm{O}, 0.4 \mathrm{~g} \mathrm{CuSO} \cdot 5 \mathrm{H}_{2} \mathrm{O}, 0.15 \mathrm{~g} \mathrm{MnSO} \mathrm{O}_{4} \cdot \mathrm{H}_{2} \mathrm{O}$, $0.1 \mathrm{~g} \mathrm{NaB} \mathrm{O}_{7} \cdot 10 \mathrm{H}_{2} \mathrm{O}, 0.05 \mathrm{~g}\left(\mathrm{NH}_{4}\right)_{6} \mathrm{Mo}_{7} \mathrm{O}_{24} \cdot 4 \mathrm{H}_{2} \mathrm{O}$, and ultra-filtrated water to $1000 \mathrm{~mL}$ [68]. All conidia were harvested in sterile, autoclaved water, then filtered through $30 \mu \mathrm{m}$ filters (MACS Milteny Biotec) and counted with a Thoma chamber.

\section{Germination assay}

Germination assay was performed by inoculating $1 \cdot 10^{6}$ spores per $m L$ in RPMI without phenol red (Thermo Fisher Scientific). At different time points pictures were taken using a Keyence BZX800 microscope and the number of germinated spores was determined by counting the ratio of spores undergoing germination (germ-tube formation) per field (100 cells).

\section{Cytotoxicity assay}

Human lung AEC A549 (ATCC-CCL 185) were maintained in F12K nutrient medium (Thermo Fisher Scientific) with the addition of $10 \%(\mathrm{v} / \mathrm{v})$ fetal bovine serum (FBS) (HyClone, GE Life science) at $37^{\circ} \mathrm{C}$ with $5 \%(\mathrm{v} / \mathrm{v}) \mathrm{CO}_{2}$. Cells of the mouse lung epithelial cell line T7 (ECACC 07021402) were maintained in F12K nutrient medium (Thermo Fisher Scientific) with the addition of $0.5 \%(\mathrm{v} / \mathrm{v})$ FBS (HyClone, GE Life science) and $0.02 \%(\mathrm{v} / \mathrm{v})$ Insulin-Transferin-Sodium Selenite (Sigma Aldrich). $2 \cdot 10^{5}$ cells per well were seeded in 24 well plate $18 h$ prior to the experiment. Before infection cells were washed once with sterile phosphate buffered saline (PBS) 1X (Gibco, Thermo Fisher Scientific) and then incubated with conidia based on the different MOls in DMEM without phenol red (Gibco, Thermo Fisher Scientific) with the addition of $10 \%(\mathrm{v} / \mathrm{v})$ FBS. A549 cells and conidia were incubated for $20 \mathrm{~h}$ at $37^{\circ} \mathrm{C}$ with $5 \%(\mathrm{v} / \mathrm{v}) \mathrm{CO}_{2}$. $\mathrm{LDH}$ release was measured using the CyQuant LDH cytotoxicity assay (Thermo Fisher Scientific) using the manufacturer instructions. The absorbance was determined using a Tecan Infinite 200 (LabX). 


\section{Isolation of alveolar epithelial type II cells and AM from mice}

A total of eighteen male and female 12-18 weeks old C57BL/6J (The Jackson Laboratory) mice were used. All animals were cared for in accordance with the European animal welfare regulation and approved by the responsible federal state authority and ethics committee in accordance with the German animal welfare act (permit no. 03-072/16).

Mice were sacrificed using $125 \mu \mathrm{L}$ ketamine per $20 \mathrm{~g}$ xylazine and the lungs were obtained as previously described [69]. After isolation, the lungs lobes were digested for $45 \mathrm{~min}$ at room temperature in $1 \mathrm{~mL}$ of dispase (Corning) and then the lung parenchyma was separated with the help of tweezers in $7 \mathrm{~mL}$ of DMEM/F12K (Thermo Fisher Scientific) containing $0.01 \mathrm{mg}$ of DNase (Sigma Aldrich). The cell suspension was first filtered twice: through a $70 \mu \mathrm{m}$ and then through a $30 \mu m$ (MACS, Miltenyi Biotec) filter and finally centrifuged. The pellet was lysed using a red blood cell (RBC) lysis buffer and re-centrifuged.

For their separation cells underwent a double magnetic labeling selection. At first, they were negatively labeled using CD45 (macrophages), CD16/32 (B/NK cells), anti-Ter (erythrocytes), CD31 (endothelial cells) (Miltyenyi Biotec) and anti-t1 $\alpha$ (alveolar epithelial type I cells) (Novus Biologicals), biotin-linked antibodies. Secondly the negative fraction was collected and positively selected for alveolar epithelial type II cells using a CD326/EpCAM antibody (eBioscience). Both labeling steps were perfomed at $4{ }^{\circ} \mathrm{C}$ for $30 \mathrm{~min}$, and followed by a second labeling with AntiBiotin Microbeads Ultrapure (Miltenyi Biotec) for $15 \mathrm{~min}$ at $4^{\circ} \mathrm{C}$. The separation was performed using an autoMACS Pro Separator machine (Miltyenyi Biotec). The final cell suspension containing type II AEC was resuspended in mouse tracheal epithelial cells (MTEC) basic medium [70]: DMEM-F12K +1\% HEPES, Na-bicarbonate, L-glutamine, penicillin-streptomycin, $0.1 \%$ amphotericin B (Gibco) supplemented with $0.001 \%$ Insulin-transferrin (Gibco), $0.1^{\mu g} / \mathrm{mL}$ cholera toxin (Sigma Aldrich), $25^{\mathrm{ng} / \mathrm{mL}}$ epidermal growth factor (Invitrogen) and mice fibroblast growth factor 7 (R\&D system), $30^{\mu g} / \mathrm{mL}_{\mathrm{L}}$ bovine pituitary extract (Gibco), 30 $\mathrm{ng} / \mathrm{mL}$ multilinear hemopoietic growth factor , $50 \mathrm{ng} / \mathrm{mL}$ human fibroblastic growth factor 10 (R\&D system), $5 \% \mathrm{FBS}, 0.01 \mu M$ retinoic acid (Sigma Aldrich) and $10 \mu M$ Rho kinase inhibitor (ROCK) (Thermo Fisher Scientific).

The isolation of AM was performed similarly, only using the first labeling step with CD45 antibodies. AM were re-suspended in MTEC basic medium with the addition of $10 \%(\mathrm{v} / \mathrm{v}) \mathrm{FBS}$.

From each mouse, a total of $1 \cdot 10^{6}$ cells were seeded onto 8 well Millicells slides (Merc, Millipore), pre-coated with $100^{\mu g} / \mathrm{mL}$ of fibronectin (Sigma Aldrich), for AEC, and incubated at $37^{\circ} \mathrm{C}$ and $5 \%(\mathrm{v} / \mathrm{v}) \mathrm{CO}_{2}$. The medium was changed every 2 days and the cells were left to rest for 7 days prior the experiment.

\section{Infection with A. fumigatus and cytokine measurement}

Alveolar epithelial type II cells were infected with $A$. fumigatus CEA10 conidia at a multiplicity of infection (MOI) of 5 , for $4,6,10$, and $24 \mathrm{~h}$. At these time points the supernatant was collected, centrifuged at $300 \cdot \mathrm{g}$ for $5 \mathrm{~min}$ and then stored at $-20^{\circ} \mathrm{C}$ for $24 \mathrm{~h}$ until measurements. The levels of interleukin (IL)-6 and tumor necrosis factor (TNF)- $\alpha$ were detected using ELISA kits (Biolegend) following the manufacturer's instructions.

\section{Acknowledgments}

We acknowledge support by the Deutsche Forschungsgemeinschaft (DFG) CRC/Transregio 124 'Pathogenic fungi and their human host: Networks of interaction' (support code 210879364) sub project B1 (J.E. and S.S.) and A1 (F.R. and A.B.). Further, C.K. acknowledges support by the 
Cluster of Excellence 'Precision Medicine in Chronic Inflammation' (support code EXC 2167) and L.R. by the Cluster of Excellence 'Balance of the Microverse' (support code EXC 2051 - Project-ID 390713860). We thank Elina Wiechens for an initial parameter search in the literature and thank Pedro Moura-Alves, MPIIB Berlin, for providing us the mouse T7 alveolar lung cells. In addition, we thank Dr. Maria Straßburger for the help with mouse experiments.

\section{References}

[1] J. P. Mizgerd, Lung infection-a public health priority, PLoS Medicine 3 (2) (2006) e76.

[2] S. Vento, F. Cainelli, Z. Temesgen, Lung infections after cancer chemotherapy, The Lancet Oncology 9 (10) (2008) 982-992.

[3] A. A. Brakhage, K. Langfelder, Menacing mold: the molecular biology of Aspergillus fumigatus, Annual Reviews in Microbiology 56 (1) (2002) 433-455.

[4] F. Tekaia, J.-P. Latgé, Aspergillus fumigatus: saprophyte or pathogen?, Current Opinion in Microbiology 8 (4) (2005) 385-392.

[5] T. R. Dagenais, N. P. Keller, Pathogenesis of Aspergillus fumigatus in invasive aspergillosis, Clinical Microbiology Reviews 22 (3) (2009) 447-465.

[6] D. H. Scharf, T. Heinekamp, N. Remme, P. Hortschansky, A. A. Brakhage, C. Hertweck, Biosynthesis and function of gliotoxin in Aspergillus fumigatus, Applied Microbiology and Biotechnology 93 (2) (2012) 467-472.

[7] J.-P. Latgé, G. Chamilos, Aspergillus fumigatus and Aspergillosis in 2019, Clinical Microbiology Reviews 33 (1).

[8] N. Osherov, Interaction of the pathogenic mold Aspergillus fumigatus with lung epithelial cells, Frontiers in Microbiology 3 (2012) 346.

[9] V. Balloy, M. Chignard, The innate immune response to Aspergillus fumigatus, Microbes and Infection 11 (12) (2009) 919-927.

[10] S. P. Brown, D. M. Cornforth, N. Mideo, Evolution of virulence in opportunistic pathogens: generalism, plasticity, and control, Trends in Microbiology 20 (7) (2012) 336-342.

[11] G. Dragonetti, M. Criscuolo, L. Fianchi, L. Pagano, Invasive Aspergillosis in Acute Myeloid Leukemia: Are We Making Progress in Reducing Mortality?, Medical Mycology 55 (1) (2017) 82-86.

[12] F. S. Taccone, A.-M. Van den Abeele, P. Bulpa, B. Misset, W. Meersseman, T. Cardoso, J.-A. Paiva, M. BlascoNavalpotro, E. De Laere, G. Dimopoulos, et al., Epidemiology of invasive aspergillosis in critically ill patients: clinical presentation, underlying conditions, and outcomes, Critical Care 19 (1) (2015) 7.

[13] M. Bartoletti, R. Pascale, M. Cricca, M. Rinaldi, A. Maccaro, L. Bussini, G. Fornaro, T. Tonetti, G. Pizzilli, E. Francalanci, et al., Epidemiology of invasive pulmonary aspergillosis among COVID-19 intubated patients: a prospective study, Clinical Infectious Diseases.

[14] H. K. Weir, T. D. Thompson, A. Soman, B. Møller, S. Leadbetter, The past, present, and future of cancer incidence in the United States: 1975 through 2020, Cancer 121 (11) (2015) 1827-1837.

[15] M. Mistry, D. Parkin, A. S. Ahmad, P. Sasieni, Cancer incidence in the United Kingdom: projections to the year 2030, British journal of cancer 105 (11) (2011) 1795.

[16] J. J. Wynn, C. E. Alexander, Increasing organ donation and transplantation: the US experience over the past decade, Transplant International 24 (4) (2011) 324-332.

[17] R. Granich, S. Gupta, B. Hersh, B. Williams, J. Montaner, B. Young, J. M. Zuniga, Trends in AIDS deaths, new infections and ART coverage in the top 30 countries with the highest AIDS mortality burden; 1990-2013, PloS One 10 (7) (2015) e0131353.

[18] M. Hoenigl, Invasive fungal disease complicating coronavirus disease 2019: when it rains, it spores, Clinical Infectious Diseases.

[19] R. J. Tanaka, N. J. Boon, K. Vrcelj, A. Nguyen, C. Vinci, D. Armstrong-James, E. Bignell, In silico modeling of spore inhalation reveals fungal persistence following low dose exposure, Scientific Reports 5 (2015) 13958.

[20] J. Pollmächer, M. T. Figge, Deciphering chemokine properties by a hybrid agent-based model of Aspergillus fumigatus infection in human alveoli, Frontiers in Microbiology 6 (2015) 503.

[21] M. Oremland, K. R. Michels, A. M. Bettina, C. Lawrence, B. Mehrad, R. Laubenbacher, A computational model of invasive aspergillosis in the lung and the role of iron, BMC Systems Biology 10 (1) (2016) 34.

[22] J. Pollmächer, S. Timme, S. Schuster, A. A. Brakhage, P. F. Zipfel, M. T. Figge, Deciphering the counterplay of Aspergillus fumigatus infection and host inflammation by evolutionary games on graphs, Scientific Reports 6 (2016) 27807. 
bioRxiv preprint doi: https://doi.org/10.1101/2021.05.12.443764; this version posted May 14, 2021. The copyright holder for this preprint (which was not certified by peer review) is the author/funder, who has granted bioRxiv a license to display the preprint in perpetuity. It is made available under aCC-BY-NC-ND 4.0 International license.

[23] M. Blickensdorf, S. Timme, M. T. Figge, Comparative Assessment of Aspergillosis by Virtual Infection Modeling in Murine and Human Lung, Frontiers in Immunology 10.

[24] J. Ewald, P. Sieber, R. Garde, S. N. Lang, S. Schuster, B. Ibrahim, Trends in mathematical modeling of hostpathogen interactions, Cellular and Molecular Life Sciences (2019) 1-14.

[25] M. Blickensdorf, S. Timme, M. T. Figge, Hybrid agent-based modeling of Aspergillus fumigatus infection to quantitatively investigate the role of pores of Kohn in human alveoli, Frontiers in Microbiology 11 (2020) 1951.

[26] B. Philippe, O. Ibrahim-Granet, M. Prevost, M. Gougerot-Pocidalo, M. S. Perez, A. Van der Meeren, J. Latge, Killing of Aspergillus fumigatus by alveolar macrophages is mediated by reactive oxidant intermediates, Infection and Immunity 71 (6) (2003) 3034-3042.

[27] D. Taramelli, M. Malabarba, G. Sala, N. Basilico, G. Cocuzza, Production of cytokines by alveolar and peritoneal macrophages stimulated by Aspergillus fumigatus conidia or hyphae, Journal of Medical and Veterinary Mycology 34 (1) (1996) 49-56.

[28] M. M. Mircescu, L. Lipuma, N. van Rooijen, E. G. Pamer, T. M. Hohl, Essential role for neutrophils but not alveolar macrophages at early time points following Aspergillus fumigatus infection, The Journal of Infectious Diseases 200 (4) (2009) 647-656.

[29] J. A. Wasylnka, M. M. Moore, Aspergillus fumigatus conidia survive and germinate in acidic organelles of A549 epithelial cells, Journal of Cell Science 116 (8) (2003) 1579-1587.

[30] V. Balloy, J.-M. Sallenave, Y. Wu, L. Touqui, J.-P. Latgé, M. Si-Tahar, M. Chignard, Aspergillus fumigatus-induced interleukin-8 synthesis by respiratory epithelial cells is controlled by the phosphatidylinositol 3-kinase, p38 MAPK, and ERK1/2 pathways and not by the toll-like receptor-MyD88 pathway, Journal of Biological Chemistry 283 (45) (2008) 30513-30521.

[31] E. Shudo, Y. Iwasa, Dynamic optimization of host defense, immune memory, and post-infection pathogen levels in mammals, Journal of Theoretical Biology 228 (1) (2004) 17-29.

[32] S. Dühring, J. Ewald, S. Germerodt, C. Kaleta, T. Dandekar, S. Schuster, Modelling the host-pathogen interactions of macrophages and Candida albicans using Game Theory and dynamic optimization, Journal of The Royal Society Interface 14 (132) (2017) 20170095.

[33] T. W. McDade, A. V. Georgiev, C. W. Kuzawa, Trade-offs between acquired and innate immune defenses in humans, Evolution, Medicine, and Public Health 2016 (1) (2016) 1-16.

[34] G. Desoubeaux, C. Cray, Rodent models of invasive aspergillosis due to Aspergillus fumigatus: still a long path toward standardization, Frontiers in Microbiology 8 (2017) 841.

[35] G. Desoubeaux, C. Cray, Animal models of aspergillosis, Comparative Medicine 68 (2) (2018) 109-123.

[36] T. Heinekamp, H. Schmidt, K. Lapp, V. Pähtz, I. Shopova, N. Köster-Eiserfunke, T. Krüger, O. Kniemeyer, A. A. Brakhage, Interference of Aspergillus fumigatus with the immune response, in: Seminars in Immunopathology, Vol. 37, Springer, 2015, pp. 141-152.

[37] B. P. Knox, A. Huttenlocher, N. P. Keller, Real-time visualization of immune cell clearance of Aspergillus fumigatus spores and hyphae, Fungal Genetics and Biology 105 (2017) 52-54.

[38] K. C. Stone, R. R. Mercer, P. Gehr, B. Stockstill, J. D. Crapo, et al., Allometric relationships of cell numbers and size in the mammalian lung, American Journal of Respiratory Cell and Molecular Biology 6 (2) (1992) 235-43.

[39] J. Knust, M. Ochs, H. J. G. Gundersen, J. R. Nyengaard, Stereological estimates of alveolar number and size and capillary length and surface area in mice lungs, The Anatomical Record: Advances in Integrative Anatomy and Evolutionary Biology: Advances in Integrative Anatomy and Evolutionary Biology 292 (1) (2009) 113-122.

[40] J. Amich, Z. Mokhtari, M. Strobel, E. Vialetto, D. Sheta, Y. Yu, J. Hartweg, N. Kalleda, K. J. Jarick, C. Brede, et al., Three-dimensional light sheet fluorescence microscopy of lungs to dissect local host immune-Aspergillus fumigatus interactions, mBio 11 (1).

[41] N. Kalleda, J. Amich, B. Arslan, S. Poreddy, K. Mattenheimer, Z. Mokhtari, H. Einsele, M. Brock, K. G. Heinze, A. Beilhack, Dynamic immune cell recruitment after murine pulmonary Aspergillus fumigatus infection under different immunosuppressive regimens, Frontiers in Microbiology 7 (2016) 1107.

[42] D. C. Sheppard, G. Rieg, L. Y. Chiang, S. G. Filler, J. E. Edwards, A. S. Ibrahim, Novel inhalational murine model of invasive pulmonary aspergillosis, Antimicrobial Agents and Chemotherapy 48 (5) (2004) 1908-1911.

[43] M. Duong, N. Ouellet, M. Simard, Y. Bergeron, M. Olivier, M. G. Bergeron, Kinetic study of host defense and inflammatory response to Aspergillus fumigatus in steroid-induced immunosuppressed mice, The Journal of Infectious Diseases 178 (5) (1998) 1472-1482.

[44] P. Borger, G. Koeter, J. Timmerman, E. Vellenga, J. Tomee, H. Kauffman, Proteases from Aspevgillus fumigatus Induce Interleukin (IL)- 6 and IL-8 Production in Airway Epithelial Cell Lines by Transcriptional Mechanisms, The Journal of Infectious Diseases 180 (4) (1999) 1267-1274.

[45] E. Cenci, A. Mencacci, A. Casagrande, P. Mosci, F. Bistoni, L. Romani, Impaired antifungal effector activity but not 
inflammatory cell recruitment in interleukin-6-deficient mice with invasive pulmonary aspergillosis, The Journal of Infectious Diseases 184 (5) (2001) 610-617.

[46] R. Araujo, A. G. Rodrigues, Variability of germinative potential among pathogenic species of Aspergillus, Journal of Clinical Microbiology 42 (9) (2004) 4335-4337.

[47] J. C. Rhodes, Aspergillus fumigatus: growth and virulence, Medical Mycology 44 (Supplement_1) (2006) S77S81.

[48] C. Paulussen, J. E. Hallsworth, S. Álvarez-Pérez, W. C. Nierman, P. G. Hamill, D. Blain, H. Rediers, B. Lievens, Ecology of aspergillosis: insights into the pathogenic potency of Aspergillus fumigatus and some other Aspergillus species, Microbial Biotechnology 10 (2) (2017) 296-322.

[49] S. S. Henriet, P. E. Verweij, A. Warris, Aspergillus nidulans and chronic granulomatous disease: a unique hostpathogen interaction, The Journal of Infectious diseases 206 (7) (2012) 1128-1137.

[50] R. W. Bastos, C. Valero, L. P. Silva, T. Schoen, M. Drott, V. Brauer, R. Silva-Rocha, A. Lind, J. L. Steenwyk, A. Rokas, et al., Functional characterization of clinical isolates of the opportunistic fungal pathogen Aspergillus nidulans, MSphere 5 (2).

[51] C. R. Bonnett, E. J. Cornish, A. G. Harmsen, J. B. Burritt, Early neutrophil recruitment and aggregation in the murine lung inhibit germination of Aspergillus fumigatus conidia, Infection and Immunity 74 (12) (2006) 65286539.

[52] E. E. Rosowski, N. Raffa, B. P. Knox, N. Golenberg, N. P. Keller, A. Huttenlocher, Macrophages inhibit Aspergillus fumigatus germination and neutrophil-mediated fungal killing, PLoS Pathogens 14 (8) (2018) e1007229.

[53] T. A. Wynn, K. M. Vannella, Macrophages in tissue repair, regeneration, and fibrosis, Immunity 44 (3) (2016) 450-462.

[54] B. Allard, A. Panariti, J. G. Martin, Alveolar macrophages in the resolution of inflammation, tissue repair, and tolerance to infection, Frontiers in Immunology 9 (2018) 1777.

[55] H. Sales-Campos, L. Tonani, C. R. B. Cardoso, M. R. V. Z. Kress, The immune interplay between the host and the pathogen in Aspergillus fumigatus lung infection, BioMed Research International 2013.

[56] V. Espinosa, A. Rivera, First line of defense: innate cell-mediated control of pulmonary aspergillosis, Frontiers in Microbiology 7 (2016) 272.

[57] R. F. Stengel, R. M. Ghigliazza, N. V. Kulkarni, Optimal enhancement of immune response, Bioinformatics 18 (9) (2002) $1227-1235$.

[58] E. Dague, D. Alsteens, J.-P. Latgé, Y. F. Dufrêne, High-resolution cell surface dynamics of germinating Aspergillus fumigatus conidia, Biophysical journal 94 (2) (2008) 656-660.

[59] K. J. Kwon-Chung, J. A. Sugui, Aspergillus fumigatus-what makes the species a ubiquitous human fungal pathogen?, PLoS pathogens 9 (12) (2013) e1003743.

[60] J. M. Cicchese, S. Evans, C. Hult, L. R. Joslyn, T. Wessler, J. A. Millar, S. Marino, N. A. Cilfone, J. T. Mattila, J. J. Linderman, et al., Dynamic balance of pro-and anti-inflammatory signals controls disease and limits pathology, Immunological Reviews 285 (1) (2018) 147-167.

[61] R. S. Malik-Sheriff, M. Glont, T. V. Nguyen, K. Tiwari, M. G. Roberts, A. Xavier, M. T. Vu, J. Men, M. Maire, S. Kananathan, et al., BioModels-15 years of sharing computational models in life science, Nucleic acids research 48 (D1) (2020) D407-D415.

[62] M. Bartl, P. Li, L. T. Biegler, Improvement of state profile accuracy in nonlinear dynamic optimization with the quasi-sequential approach, AIChE Journal 57 (8) (2011) 2185-2197.

[63] F. Wessely, M. Bartl, R. Guthke, P. Li, S. Schuster, C. Kaleta, Optimal regulatory strategies for metabolic pathways in Escherichia coli depending on protein costs, Molecular Systems Biology 7 (1).

[64] M. Bartl, M. Kötzing, S. Schuster, P. Li, C. Kaleta, Dynamic optimization identifies optimal programmes for pathway regulation in prokaryotes, Nature Communications 4 (2013) 2243.

[65] M. Bartl, M. Pfaff, A. Ghallab, D. Driesch, S. G. Henkel, J. G. Hengstler, S. Schuster, C. Kaleta, R. Gebhardt, S. Zellmer, et al., Optimality in the zonation of ammonia detoxification in rodent liver, Archives of Toxicology 89 (11) (2015) 2069-2078.

[66] J. Ewald, M. Bartl, C. Kaleta, Deciphering the regulation of metabolism with dynamic optimization: an overview of recent advances, Biochemical Society Transactions 45 (4) (2017) 1035-1043.

[67] E. A. Groen, E. A. Bokkers, R. Heijungs, I. J. de Boer, Methods for global sensitivity analysis in life cycle assessment, The International Journal of Life Cycle Assessment 22 (7) (2017) 1125-1137.

[68] A. A. Brakhage, J. Van den Brulle, Use of reporter genes to identify recessive trans-acting mutations specifically involved in the regulation of Aspergillus nidulans penicillin biosynthesis genes., Journal of Bacteriology 177 (10) (1995) 2781-2788.

[69] M. Sinha, C. A. Lowell, Isolation of highly pure primary mouse alveolar epithelial type II cells by flow cytometric 
cell sorting, Bio-protocol 6 (22).

[70] Y. You, E. J. Richer, T. Huang, S. L. Brody, Growth and differentiation of mouse tracheal epithelial cells: selection of a proliferative population, American Journal of Physiology-Lung Cellular and Molecular Physiology 283 (6) (2002) L1315-L1321.

[71] M. Bertuzzi, M. Schrettl, L. Alcazar-Fuoli, T. C. Cairns, A. Muñoz, L. A. Walker, S. Herbst, M. Safari, A. M. Cheverton, D. Chen, et al., The pH-responsive PacC transcription factor of Aspergillus fumigatus governs epithelial entry and tissue invasion during pulmonary aspergillosis, PLoS Pathogens 10 (10) (2014) e1004413.

[72] M. Saffarzadeh, C. Juenemann, M. A. Queisser, G. Lochnit, G. Barreto, S. P. Galuska, J. Lohmeyer, K. T. Preissner, Neutrophil extracellular traps directly induce epithelial and endothelial cell death: a predominant role of histones, PloS One 7 (2) (2012) e32366.

[73] S. Hirano, Interaction of rat alveolar macrophages with pulmonary epithelial cells following exposure to lipopolysaccharide, Archives of Toxicology 70 (3-4) (1996) 230-236.

[74] A. Alflen, S. Prüfer, K. Ebner, S. Reuter, P. A. Lopez, I. Scharrer, F. Banno, M. Stassen, H. Schild, K. Jurk, et al., ADAMTS-13 regulates neutrophil recruitment in a mouse model of invasive pulmonary aspergillosis, Scientific Reports 7 (1) (2017) 7184.

[75] T. T. Ng, G. D. Robson, D. W. Denning, Hydrocortisone-enhanced growth of Aspergillus spp.: implications for pathogenesis, Microbiology 140 (9) (1994) 2475-2479.

[76] S. Slesiona, M. Gressler, M. Mihlan, C. Zaehle, M. Schaller, D. Barz, B. Hube, I. D. Jacobsen, M. Brock, Persistence versus escape: Aspergillus terreus and Aspergillus fumigatus employ different strategies during interactions with macrophages, PloS One 7 (2) (2012) e31223.

[77] A. J. Thorley, P. A. Ford, M. A. Giembycz, P. Goldstraw, A. Young, T. D. Tetley, Differential regulation of cytokine release and leukocyte migration by lipopolysaccharide-stimulated primary human lung alveolar type II epithelial cells and macrophages, The Journal of Immunology 178 (1) (2007) 463-473.

[78] M. Röhm, M. J. Grimm, A. C. D'Auria, N. G. Almyroudis, B. H. Segal, C. F. Urban, NADPH oxidase promotes neutrophil extracellular trap formation in pulmonary aspergillosis, Infection and Immunity 82 (5) (2014) 17661777. 


\section{Appendix A. Parameter estimation}

\section{Documentation of parameter estimation and calculation}

Average number of AEC (type I and II) per alveolus. The total number of AEC in several mammalian species was elucidated by Stone et al. [38] and reports for murine lungs $11.6+$ $14.8=26.4 \cdot 10^{6}$ cells (type I and II). Knust et al. [39] determined $2.31 \cdot 10^{6}$ alveoli for mice with a comparable weight $(20 \mathrm{~g})$ and we estimate $L(0)=L_{0}=\frac{26.4}{2.31}=11.43$ cells.

$l_{1}$ Rate of hyphae destroying AEC. In [71], epithelial damage was assessed in mice by LDH release in bronchial lavage showing a 2.5 fold change of damage after $24 \mathrm{~h}$. The rate is calculated by $l_{1}=\frac{\ln (\text { fold change })}{.24 h}=0.0382$.

$l_{2}$ Rate of neutrophils damaging AEC. In [72], neutrophils were stimulated in vivo with LPS and cell damage in lungs was assessed as free elastase activity in BALF. The relative damage was $72 \%$ after $12 \mathrm{~h}$ and we estimate $l_{2}=\frac{\ln (1-0.72)}{12 h}=0.1061$

$l_{3}$ As $l_{2}$ but for AM. Host damage mediated by AM is less frequently measured and we therefore rely on data of Hirano [73]. In that study rat epithelial cells (SV40T2) were co-cultured with rat alveolar macrophages and stimulated by LPS. The damage was determined by transepithelial resistance and dropped from $11 \Omega \mathrm{cm}^{2}$ to $0.5 \Omega \mathrm{cm}^{2}$ within $24 \mathrm{~h}$. To this end, we calculate $l_{3}=\frac{\ln \left(\frac{11}{0.5}\right)}{24 h}$.

(0), $R_{0}$ Initial dose of resting conidia. Value depends on the scenarios. In vivo experiments with mice typically challenged with $10^{5}-10^{7}$ conidia. Therefore, alveolus occupation is typically 0.1 - 10 conidia per alveolus. A detailed calculation is also provided by Blickensdorf et al. [23].

$r_{1}$ Average time for swelling of conidia. After $3 \mathrm{~h}$ the conidial rodlet layer is disrupted [58].

$s_{1}$ Average time for germination of swollen conidia. Total time from resting conidia to germination is on average $7 \mathrm{~h}$ [59]. Therefore $s_{1}=7 h-r_{1}=4 h$.

$s_{2}$ Killing rate of swollen conidia by AM. Intensively studied, but rates show high variation across studies [26]. In contrast, the killing rate of conidia by AEC is more rarely calculated. However, in [29] both was investigated. Accordingly, murine AM showed faster killing than AEC along all timepoints. For murine AM, conidia survival was $0.6 \%$ after $12 \mathrm{~h}$ of interaction and we calculate $s_{2}=\frac{\ln (0.006)}{12 h}=0.4263$.

$s_{3}$ Killing rate of swollen conidia by neutrophils. Phagocytosis and killing rate of conidia by neutrophils have been frequently studied. However, in the study of Alflen et al. [74] the killing rate of conidia and hyphae was compared and murine neutrophils were used. Conidial viability dropped to $39 \%$ after $4 \mathrm{~h}$ of co-incubation and we calculate $s_{3}=-\frac{0.39}{4 h}=0.2354$.

$s_{4}$ Killing rate of swollen conidia by AEC. From the same study as for $s_{2}$ [29], AEC possessed a slower killing rate and we calculate $s_{2}=\frac{\ln (0.065)}{12 h}=0.2278$. 
$h_{1}$ Growth rate of hyphae. The increase of hyphal length with time is a good estimator for doubling times of cells since cell size is rather constant. In glucose medium and at $37^{\circ} \mathrm{C}$ the length of hyphae doubles every $1 \mathrm{~h}$ [75]. The rate is then $h_{1}=\frac{\ln (2}{1 h}=0.6533$.

$h_{2}$ Killing rate of hyphae by neutrophils. From the same study as used to estimate $s_{3}$ [74], the viability of hyphae was measured after co-incubation with neutrophils. After $4 \mathrm{~h} 68 \%$ of initial fungal cells were viable, which equals a rate of $-\frac{0.68}{4 h}=0.0964$. However, hyphae are growing at the same time and therefore we estimate $h_{2}=h_{1}+0.0964$ since neutrophil killing outpaces hyphal growth by the above calculated rate.

$M(0)$ Number of resident AM per alveolus in an uninfected host. Recently, Amich et al. [40] were able to use 3D light sheet fluorescence microscopy to investigate invasive aspergillosis in in vivo mouse models. Due to this imaging approach one can precisely determine the number of immune cells within an alveolus. On average they find $M(0)=0.2926$ AM per alveolus. Note, that in the agent-based model of Blickensdorf et al. [23] a slightly higher number of AM per murine alveolus (0.74) was calculated based on whole tissue cell counts [38].

$m_{1}$ Rate of recruitment of AM by influx and maturation of monocytes. To complement data used for $M(0)$ and $N(0)$, we estimate the recruitment rates of AM and neutrophils based on data from a previous publication [41] of the same group of authors, which performed 3D light sheet fluorescence microscopy [40]. In that publication a similar murine infection model of invasive aspergillosis was used, but cell numbers were quantified by fluorescence-activated cell sorting (FACS) of lung tissue samples. For AM a rise from 2.75 to $910^{5}$ cells in $4 \mathrm{~h}$ after a mild dose of conidia was reported. We estimate $m_{1}=M(0) \frac{9}{2.75 \cdot 4 h}$.

$m_{2}$ Rate of alveolar macrophage depletion. Based on the same study as $m_{1}$ [41] macrophage level was on control level after $12 \mathrm{~h}$ of peak inflammation. So we calculate $m_{2}=M(0) \frac{9}{2.75 \cdot 12 \mathrm{~h}}$.

$m_{3}$ Lysis rate of AM by swollen conidia. While phagocytosis and killing rate by AM are frequently reported the vice versa scenario is rarely depicted. For mice cytotoxicity of swollen conidia was reported [76] to be $84 \%$ after $24 \mathrm{~h}$ that corresponds to a rate of $m_{3}=-\frac{\ln (1-0.84)}{.24}=$ 0.0764 .

$c_{1}$ Release rate of pro-inflammatory cytokines by AM. We intentionally do not model a single cytokine due to the complex network of cytokine functions and signaling to recruit neutrophils. Based on several studies investigating the time course of prominent pro-inflammatory cytokines we decided to set the cytokine release rate to $c_{1}=1$ to mimic the peak in cytokine concentration at 5-10h after infection or stimulation with LPS.

$c_{2}$ Release rate of pro-inflammatory cytokines by lung AEC. Cytokine release of lung AEC is not well understood and only covered by few studies. A pioneering study [77] investigated the potential of human lung AM and AEC to recruit neutrophils when stimulated by LPS. Since AEC showed a $43.75 \%$ higher number of recruited neutrophils under similar conditions, we set $c_{2}=1.14375$.

$c_{3}$ Decay rate of pro-inflammatory cytokines. As for $c_{1}$ we estimated the decay rate based on the investigation of several time courses and observed that decay is slower than release. For reference we set $c_{3}=0.1$. 
$N(0)$ Number of tissue resident neutrophils. As described for $M(0)$ microscopy data of murine lungs reported a neutrophil density of $N(0)=0.2628$ neutrophils per alveolus [40].

$n_{1}$ Rate of neutrophil recruitment by influx of leukocytes. Similar to the definition of $m_{1}$ an increase of neutrophils is reported in [41] from $1.9 \cdot 10^{5} \mathrm{cells}$ to $7.6 \cdot 10^{5} \mathrm{cells}$. To this end, we calculate $n_{1}=N(0) \frac{7.6 \text {. }}{1.9 \cdot 4 h}$.

$n_{2}$ Rate of neutrophil depletion. Based on the same study as $n_{1}$ [41] neutrophil level was on control level after $12 \mathrm{~h}$ of peak inflammation. So we calculate $n_{2}=N(0) \frac{7.6}{2.8 \cdot 12 \mathrm{~h}}$.

$n_{3}$ Lysis rate of neutrophils by fungal cells. In [78] induced decondensation of nuclei of neutrophils $\left(1 \cdot 10^{6}\right.$ cells $)$ was measured in response to short hyphae $(750 \mathrm{CFU}$ with length of $10-100 \mu \mathrm{m})$. After $6 \mathrm{~h}$ of co-incubation $28 \%$ of neutrophil nuclei were decondensed. Based on the assumption that hyphae contain around 10 nuclei at the reported length, we estimate an MOI of $1: 10$ and calculate the rate as $n_{3}=-\frac{\ln (1-0.28)}{0.1 \cdot 6 h}$.

\section{Appendix B. SBML model description}

The described model of invasive aspergillosis is provided as SBML and COPASI file. To enable simulation, control variables $u_{1-4}$ are fixed. Further, the model is archived in EBI BioModels under the accession MODEL2105110001. 
bioRxiv preprint doi: https://doi org/10.1101/2021.05.12.443764. this version posted May 14, 2021. The copyright holder for this preprint (which was not certified by peer review) is the author/funder, who has granted bioRxiv a license to display the preprint in perpetuity. It is made available under aCC-BY-NC-ND 4.0 International license.

\section{Appendix C. Influence of weighting innate immune response objectives}

Model behavior with no fungal burden minimization and only minimization of tissue damage.
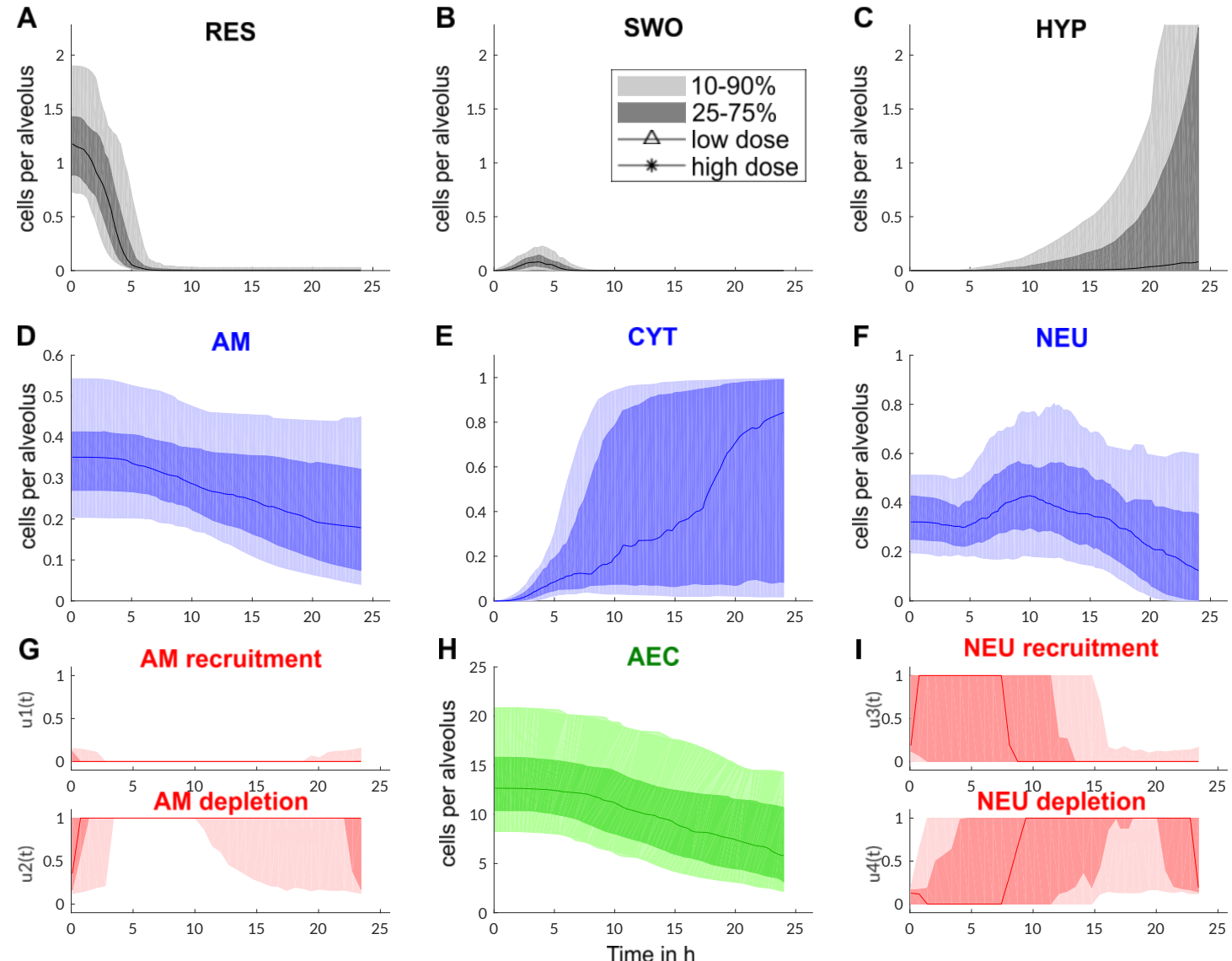

Figure C.7: Dynamics of innate immune response of the murine host challenged with a high dose of conidia (one per alveolus). The solution space of 100 parameter sets is depicted with shadings indicating the confidence intervals of time courses. Colors as in previous figures and equations. 
bioRxiv preprint doi: https://doi org/10.1101/2021.05.12.443764; this version posted May 14, 2021. The copyright holder for this preprint (which was not certified by peer review) is the author/funder, who has granted bioRxiv a license to display the preprint in perpetuity. It is made available under aCC-BY-NC-ND 4.0 International license.

Model behavior with only fungal burden minimization and no minimization of tissue damage.
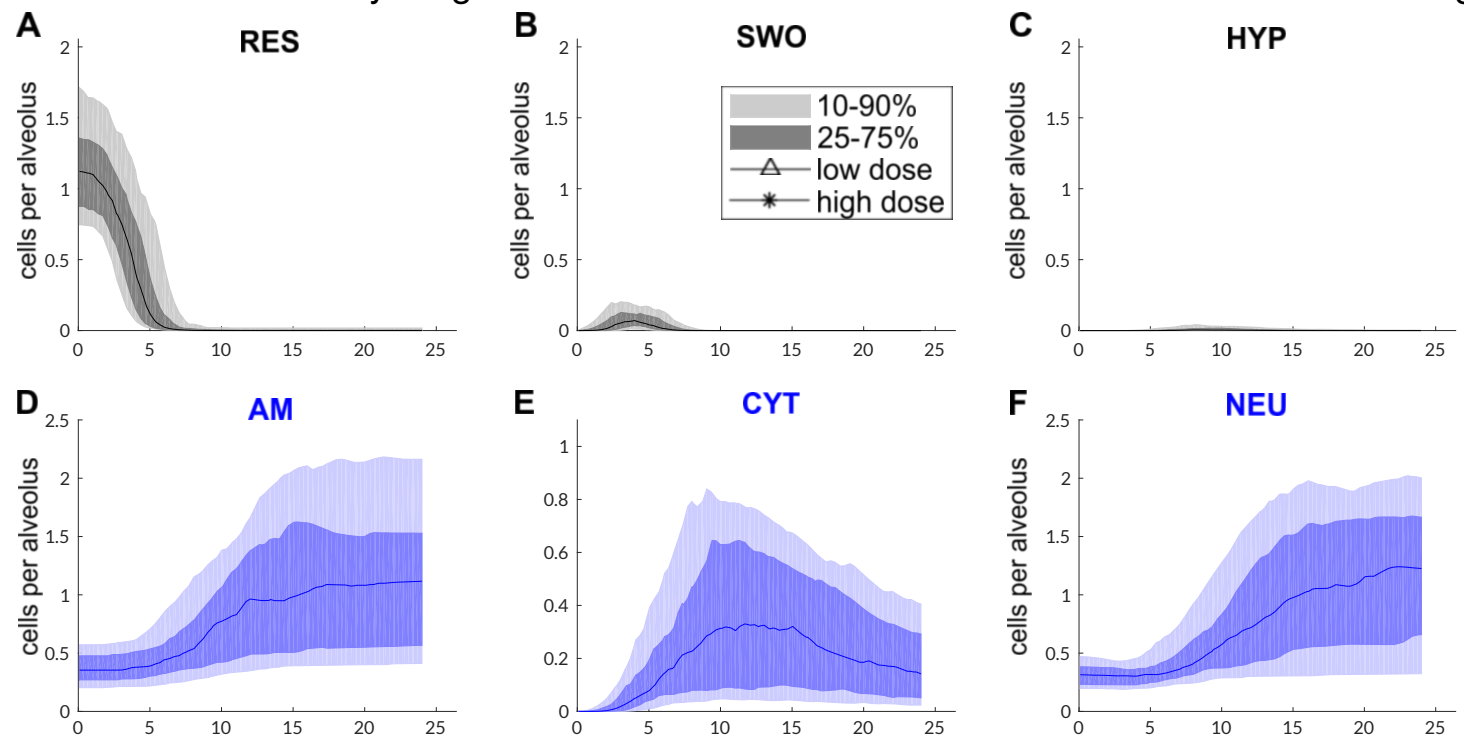

E

CYT
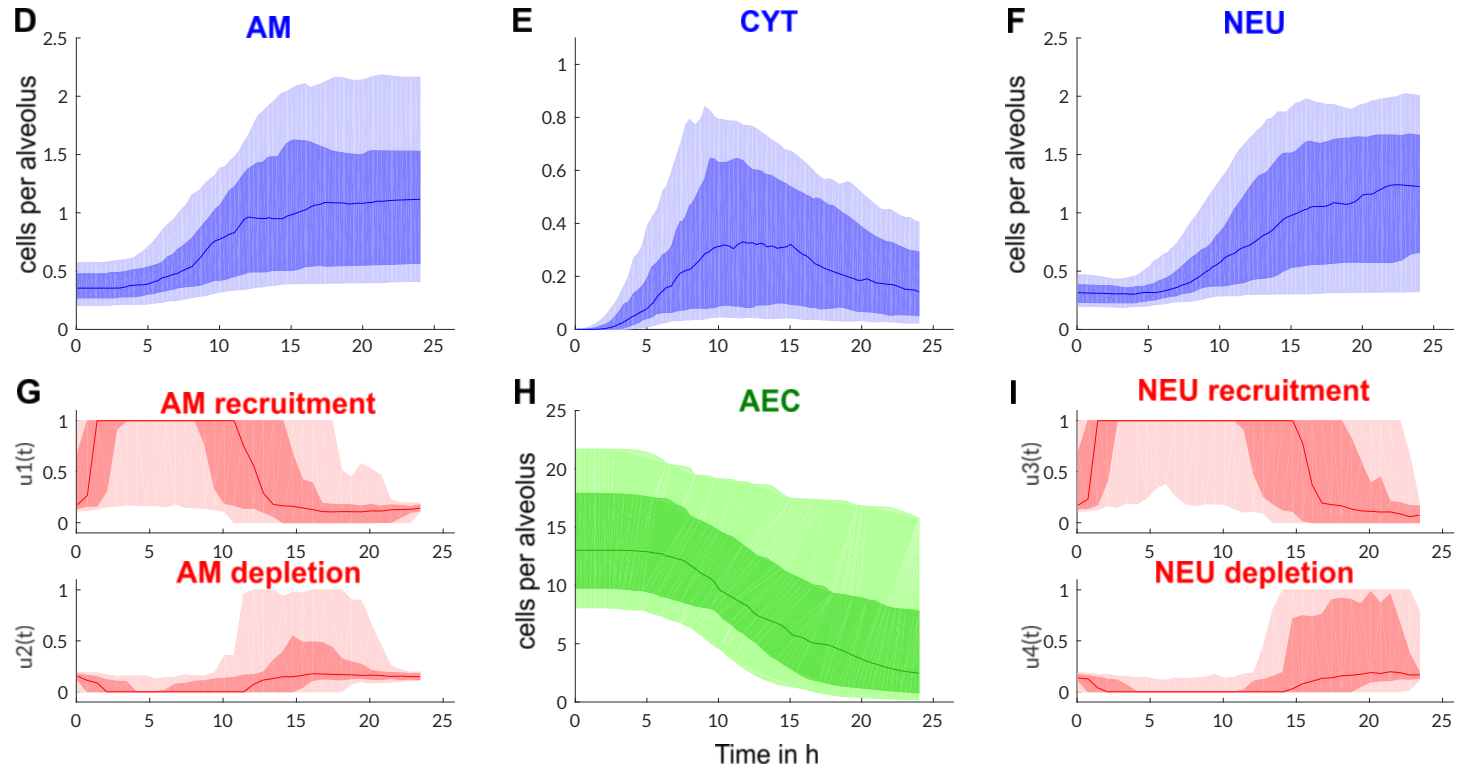

Figure C.8: Dynamics of innate immune response of the murine host challenged with a high dose of conidia (one per alveolus). The solution space of 100 parameter sets is depicted with shadings indicating the confidence intervals of time courses. Colors as in previous figures and equations. 
bioRxiv preprint doi: https://doi.org/10.1101/2021.05.12.443764; this version posted May 14, 2021. The copyright holder for this preprint (which was not certified by peer review) is the author/funder, who has granted bioRxiv a license to display the preprint in perpetuity. It is made available under aCC-BY-NC-ND 4.0 International license.

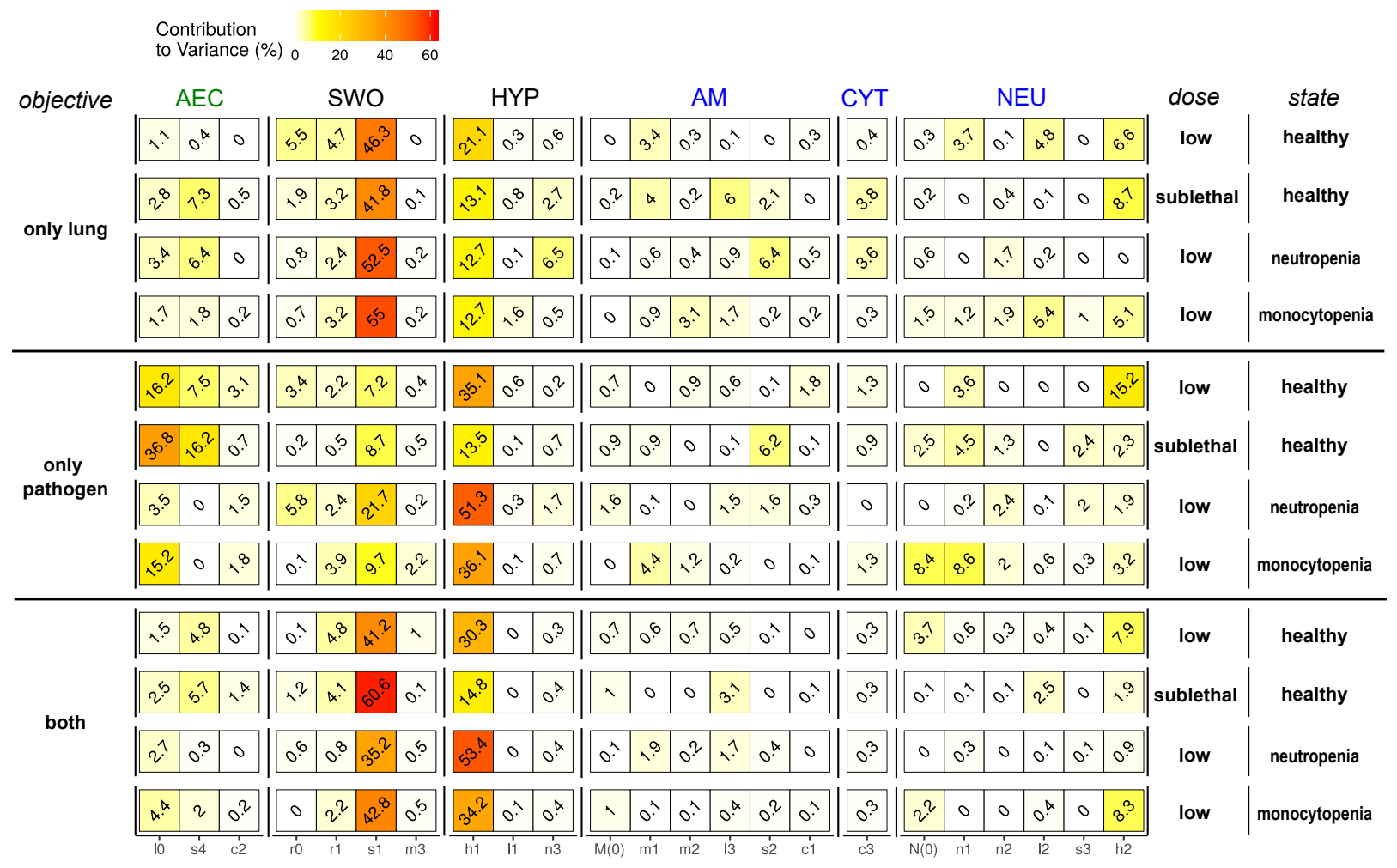

Figure C.9: Influence of parameters on the severeness of infection depicted by the contribution to variance (brown). This relative contribution is based on Spearman rank correlation of parameter value and objective value of the optimal solution. 


\section{Appendix D. Influence of MOI on cytotoxicity of Aspergillus spp. against epithelial cells}
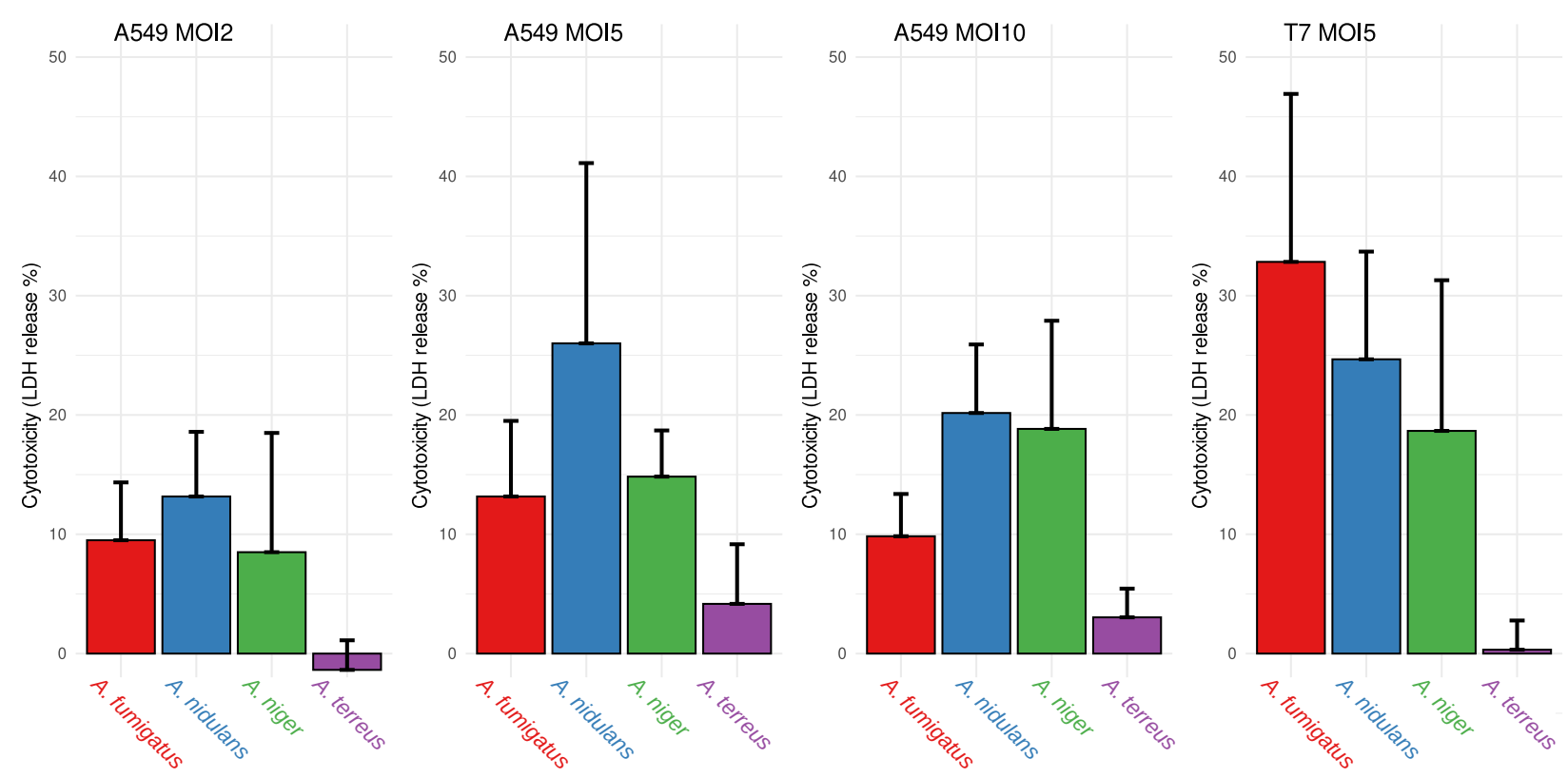

Figure D.10: In addition to the depicted LDH release measurements of epithelial cells upon 24h co-incubation with Aspergillus spp. at an $M O I=5$ (main text Figure 4P and E), cytotoxicity was measured for $M O I=2$ and $M O I=10$ for human A549 cells to demonstrate the influence of fungal burden. 